\title{
Relativistic Gauge Invariant Wave Equation of the Electron-Neutrino
}

\author{
Claude Daviau1, Jacques Bertrand ${ }^{2}$ \\ ${ }^{1}$ Le Moulin de la Lande, Pouillé-les-Coteaux, France \\ ${ }^{2} 15$ Avenue Danielle Casanova, Saint-Gratien, France \\ Email: claude.daviau@nordnet.fr, bertrandjacques-m@orange.fr
}

Received 29 March 2014; revised 22 April 2014; accepted 14 May 2014

Copyright (C) 2014 by authors and Scientific Research Publishing Inc.

This work is licensed under the Creative Commons Attribution International License (CC BY).

http://creativecommons.org/licenses/by/4.0/

c) (i) Open Access

\begin{abstract}
With the right and the left waves of an electron, plus the left wave of its neutrino, we write the tensorial densities coming from all associations of these three spinors. We recover the wave equation of the electro-weak theory. A new non linear mass term comes out. The wave equation is form invariant, then relativistic invariant, and it is gauge invariant under $U(1) \times S U(2)$, Lie group of electro-weak interactions. The invariant form of the wave equation has the Lagrangian density as real scalar part. One of the real equations equivalent to the invariant form is the law of conservation of the total current.
\end{abstract}

\section{Keywords}

Invariance Group, Dirac Equation, Weak Interactions, Gauge Invariance, Electron, Neutrino, Clifford Algebras, Magnetic Monopole

\section{Introduction}

The standard model of quantum physics uses the Dirac equation for the wave of the electron and the wave of the electronic neutrino. Weak interactions mix the left wave of the electron and the left wave of its neutrino. We start from the rewrite of this part of the standard model in the Clifford algebra ${ }^{1}$ of space-time [1]. We use a nonlinear homogeneous wave equation which has the Dirac equation as linear approximation. This wave equation has been extended to account for the electro-weak gauge group. It is a wave equation for both the right and left spinors of the electron and for the left spinor of the electronic neutrino. The wave is then a function of space and time with 12 real parameters.

${ }^{1} \mathrm{~A}$ complete introduction to this mathematical tool was made in [1]-[5]. 
We got with the 8 real parameters of the wave of the electron [4] $36=8 \times 9 / 2$ tensorialdensities ${ }^{2}$. Now with 12 real parameters $78=12 \times 13 / 2$ tensorial densities are awaited. We shall need most of them, so the first part of this study reviews these 78 densities.

In the case of the electron alone the wave equation comes from a Lagrangian density and this is true both with the nonlinear homogeneous equation and with its linear approximation. The difference between these two densities is in the mass term. We got an invariant form for both equations. Since there are 8 real parameters, the wave equation is equivalent to 8 equations. The real one is the cancellation of the Lagrangian density, and another one is the conservation of the current of probability.

It is well known that the electro-weak theory has a problem with the mass term of the Dirac equation, because this term links the left spinor and the right spinor that behave differently in the electro-weak gauge. Then this part of the standard model begins with an electron without mass term and a complicated model of symmetry spontaneously broken is necessary to get a mass term. The aim of this article is to study a wave equation with a mass term that is both relativistic invariant and gauge invariant under the electro-weak gauge group. This was previously thought as impossible.

An invariant form of this wave equation also exists; the Lagrangian density is the real part of this invariant form. We get the wave equation by the Lagrangian mechanism. The conservation of the total current is one ofthe numeric equations equivalent to the invariant form. The Dirac equation with mass term is the linear approximation of our wave equation for electron + neutrino when we cancel the wave of the neutrino.

\section{Tensors}

Each of the three spinors has 4 parameters, and then each gives $4 \times 5 / 2=10$ components of tensors, a spacetime vector (4 components) and a space-time bivector (6 components).

With the only right spinor $\phi_{R}$ of the electron

$$
\phi_{R}=\sqrt{2}\left(\begin{array}{ll}
\xi_{1} & 0 \\
\xi_{2} & 0
\end{array}\right)
$$

we get the space-time vector $D_{R}$ and the space-time bivector $S_{R}$ satisfying ${ }^{3}$

$$
D_{R}=\phi_{R} \phi_{R}^{\dagger} ; S_{R}=\phi_{R} \sigma_{1} \bar{\phi}_{R} .
$$

$D_{R}$ is a space-time vector, because it satisfies $D_{R}^{\dagger}=D_{R}$.

Similarly with the left spinor $\phi_{L}$ of the electron

$$
\phi_{L}=\sqrt{2}\left(\begin{array}{cc}
0 & -\eta_{2}^{*} \\
0 & \eta_{1}^{*}
\end{array}\right) ; \phi_{e}=\phi_{R}+\phi_{L}
$$

we get the space-time vector $D_{L}$ and the space-time bivector $S_{L}$ satisfying

$$
D_{L}=\phi_{L} \phi_{L}^{\dagger} ; S_{L}=\phi_{L} \sigma_{1} \bar{\phi}_{L} \text {. }
$$

It is well known [6] that these currents $D_{R}, D_{L}$ are the fundamental ones in the Dirac theory, and the usual currents $J=D_{0}$ and $K=D_{3}$ are sum and difference of these chiral currents

$$
D_{0}=D_{R}+D_{L} ; D_{3}=D_{R}-D_{L} .
$$

With the left spinor $\phi_{n}$ of the electronic neutrino

$$
\phi_{n}=\sqrt{2}\left(\begin{array}{cc}
0 & -\zeta_{2}^{*} \\
0 & \zeta_{1}^{*}
\end{array}\right)
$$

we get the space-time vector $D_{n}$ and the space-time bivector $S_{n}$ satisfying

$$
D_{n}=\phi_{n} \phi_{n}^{\dagger} ; \quad S_{n}=\phi_{n} \sigma_{1} \bar{\phi}_{n} .
$$

Next with two of these three spinors we can get 16 densities. We begin with $\phi_{R}$ and $\phi_{L}$. We let

\footnotetext{
${ }^{2}$ Only 16 were known with the Dirac formalism.

${ }^{3}$ A detailed calculation of components is in Appendix A.
} 


$$
\begin{aligned}
& P=2 \phi_{R} \bar{\phi}_{L}=a+S_{R L} ; \bar{P}=2 \phi_{L} \bar{\phi}_{R}=a-S_{R L} \\
& I=D_{R L}+i d_{R L}=2 \phi_{R} \sigma_{1} \phi_{L}^{\dagger} ; I^{\dagger}=D_{R L}-i d_{R L}=2 \phi_{L} \sigma_{1} \phi_{R}^{\dagger}
\end{aligned}
$$

$a$ and $S_{R L}$ are known in the Dirac frame:

$$
a=\operatorname{det}\left(\phi_{e}\right)=\Omega_{1}+i \Omega_{2}=\rho_{e} \mathrm{e}^{i \beta}=\phi_{e} \bar{\phi}_{e}=2\left(\xi_{1} \eta_{1}^{*}+\xi_{2} \eta_{2}^{*}\right)
$$

where $\Omega_{1}, \Omega_{2}$ are the relativistic invariants of the Dirac theory, and $\beta$ is the Yvon-Takabayasi angle [1] [7]. $S_{3}=S_{R L}$ [5] is the bivector which, with $\Omega_{1}, \Omega_{2}, D_{0}$ and $D_{3}$, gives the 16 components of tensors known in the complex Dirac theory. They are those invariant under the electric gauge [3]. $D_{R L}=D_{1}$ and $d_{R L}=D_{2}$ are space-time vectors ${ }^{4}$. To see this, we can consider the form invariance of the Dirac theory. $M$ being any complex $2 \times 2$ matrix, the transformation from the space-time in to itself, which to any $x=\left(\begin{array}{cc}x^{0}+x^{3} & x^{1}-i x^{2} \\ x^{1}+i x^{2} & x^{0}-x^{3}\end{array}\right)$ associates $x^{\prime}$ satisfying

$$
x^{\prime}=\left(\begin{array}{cc}
x^{\prime 0}+x^{\prime 3} & x^{\prime 1}-i x^{\prime 2} \\
x^{\prime 1}+i x^{\prime 2} & x^{\prime 0}-x^{\prime 3}
\end{array}\right)=R(x)=M x M^{\dagger}
$$

is [4] [5] [8] a Lorentz dilation, product of a Lorentz rotation and of a homothety with ratio $r$ satisfying

$$
M \bar{M}=\operatorname{det}(M)=r \mathrm{e}^{\mathrm{i} \theta}, r=|\operatorname{det}(M)| .
$$

We get

$$
\begin{aligned}
& \phi_{e}^{\prime}=M \phi_{e} ; \phi_{n}^{\prime}=M \phi_{n} \\
& D_{R L}^{\prime}=\phi_{R}^{\prime} \sigma_{1} \phi_{L}^{\prime \dagger}+\phi_{L}^{\prime} \sigma_{1} \phi_{R}^{\prime \dagger}=M \phi_{R} \sigma_{1} \phi_{L}^{\dagger} M^{\dagger}+M \phi_{L} \sigma_{1} \phi_{R}^{\dagger} M^{\dagger}=M D_{R L} M^{\dagger} .
\end{aligned}
$$

So $D_{R L}$ is a quantity varying like $x$, a contravariant vector. It is the same for $D_{R}, D_{L}, D_{n}$ or $d_{R L}$. We get also

$$
a^{\prime}=M \phi_{e} \bar{\phi}_{e} \bar{M}=M a \bar{M}=a M \bar{M}=r \mathrm{e}^{\mathrm{i} \theta} a .
$$

Then

$$
a^{\prime} a^{\prime *}=r \mathrm{e}^{i \theta} \operatorname{are}^{-i \theta} a^{*}=r^{2} a a^{*}
$$

Now with $\phi_{L}$ and $\phi_{n}$ we let

$$
\begin{aligned}
& P=2 \hat{\phi}_{n} \sigma_{1} \phi_{L}^{\dagger}=b+S_{L n} ; \quad \bar{P}=2 \hat{\phi}_{L} \bar{\sigma}_{1} \phi_{n}^{\dagger}=b-S_{L n} \\
& I=D_{L n}+i d_{L n}=2 \phi_{n} \phi_{L}^{\dagger} ; \quad I^{\dagger}=D_{L n}-i d_{L n}=2 \phi_{L} \phi_{n}^{\dagger}
\end{aligned}
$$

Vectors $D_{L n}$ and $d_{L n}$ are contravariant vectors, $S_{L n}$ is a bivector. We shall need

$$
\begin{aligned}
& b=\hat{\phi}_{n} \sigma_{1} \phi_{L}^{\dagger}+\hat{\phi}_{L} \bar{\sigma}_{1} \phi_{n}^{\dagger}=2\left(\eta_{1} \zeta_{2}-\eta_{2} \zeta_{1}\right) \\
& b^{\prime}=\hat{M} b M^{\dagger}=\hat{M} M^{\dagger} b=r \mathrm{e}^{-i \theta} b ; b^{\prime} b^{\prime *}=r^{2} b b^{*} .
\end{aligned}
$$

Finally with $\phi_{R}$ and $\phi_{n}$ we let

$$
\begin{aligned}
& P=2 \phi_{R} \bar{\phi}_{n}=c+S_{R n} ; \quad \bar{P}=2 \phi_{n} \bar{\phi}_{R}=c-S_{R n} \\
& I=D_{R n}+i d_{R n}=2 \phi_{R} \sigma_{1} \phi_{n}^{\dagger} ; \quad I^{\dagger}=D_{R n}-i d_{R n}=2 \phi_{n} \sigma_{1} \phi_{R}^{\dagger}
\end{aligned}
$$

Vectors $D_{R n}$ and $d_{R n}$ are contravariant vectors, $S_{R n}$ is a bivector. We shall need

$$
\begin{aligned}
& c=\phi_{R} \bar{\phi}_{n}+\phi_{n} \bar{\phi}_{R}=2\left(\xi_{1} \zeta_{1}^{*}+\xi_{2} \zeta_{2}^{*}\right) \\
& c^{\prime}=M b \bar{M}=M \bar{M} c=r \mathrm{e}^{i \theta} c ; \quad c^{\prime} c^{\prime *}=r^{2} c c^{*} .
\end{aligned}
$$

The main invariant term of the electron wave is [1] $m \rho$. Since we get now not only one, but three similar ${ }^{4}$ If $A=\left(\begin{array}{cc}a & -d^{*} \\ b & c^{*}\end{array}\right)$ we get $\hat{A}=\left(\begin{array}{cc}c & -b^{*} \\ d & a^{*}\end{array}\right), \quad \bar{A}=\hat{A}^{\dagger}$ and $A^{+}=\left(\begin{array}{cc}a^{*} & b^{*} \\ -d & c\end{array}\right)$. 
terms, the natural generalization to the wave of the electron and its neutrino is ${ }^{5}$

$$
\begin{aligned}
& \rho=\sqrt{a a^{*}+b b^{*}+c c^{*}} \\
& m \rho=m^{\prime} r \rho=m^{\prime} \rho^{\prime} .
\end{aligned}
$$

\section{Getting the Wave Equation}

Since this invariant term is the generalization of the invariant mass term of the electron wave, since this term is the mass term of the Lagrangian density [1], the Lagrangian density of the wave of the electron and its neutrino is the real scalar part $\mathcal{L}=\langle L\rangle$ of

$$
L=\tilde{\Psi} \mathbf{D} \Psi \gamma_{012}+m \rho ; \Psi=\left(\begin{array}{cc}
\phi_{e} & \phi_{n} \\
\hat{\phi}_{n} & \hat{\phi}_{e}
\end{array}\right)
$$

where $\gamma_{012}=\gamma_{0} \gamma_{1} \gamma_{2}, \tilde{\Psi}$ is the reverse and $\mathbf{D}$ is the covariant derivative satisfying

$$
\mathbf{D}=\partial+\frac{g_{1}}{2} \mathbf{B} P_{0}+\frac{g_{2}}{2}\left(\mathbf{W}^{1} P_{1}+\mathbf{W}^{2} P_{2}+\mathbf{W}^{3} P_{3}\right) ; \quad \tilde{\Psi}=\left(\begin{array}{ll}
\bar{\phi}_{e} & \phi_{n}^{\dagger} \\
\bar{\phi}_{n} & \phi_{e}^{\dagger}
\end{array}\right) .
$$

Projectors $P_{\mu}$ satisfy

$$
\begin{aligned}
& P_{ \pm}(\Psi)=\frac{1}{2}\left(\Psi \pm \mathbf{i} \Psi \gamma_{21}\right) ; \mathbf{i}=\gamma_{0123} \\
& P_{0}(\Psi)=\Psi \gamma_{21}+\frac{1}{2} \Psi \mathbf{i}+\frac{1}{2} \mathbf{i} \Psi \gamma_{30}=\Psi \gamma_{21}+P_{-}(\Psi) \mathbf{i} \\
& P_{1}(\Psi)=\frac{1}{2}\left(\mathbf{i} \Psi \gamma_{0}+\Psi \gamma_{012}\right)=P_{+}(\Psi) \gamma_{3} \mathbf{i} \\
& P_{2}(\Psi)=\frac{1}{2}\left(\Psi \gamma_{3}-\mathbf{i} \Psi \gamma_{123}\right)=P_{+}(\Psi) \gamma_{3} \\
& P_{3}(\Psi)=\frac{1}{2}\left(-\Psi \mathbf{i}+\mathbf{i} \Psi \gamma_{30}\right)=P_{+}(\Psi)(-\mathbf{i}) .
\end{aligned}
$$

Noting $P_{\mu} P_{v}(\Psi)=P_{\mu}\left[P_{v}(\Psi)\right]$ they satisfy

$$
\begin{aligned}
& P_{1} P_{2}=P_{3}=-P_{2} P_{1} \\
& P_{2} P_{3}=P_{1}=-P_{3} P_{2} \\
& P_{3} P_{1}=P_{2}=-P_{1} P_{3} \\
& P_{1}^{2}=P_{2}^{2}=P_{3}^{2}=-P_{+} \\
& P_{0} P_{j}=P_{j} P_{0}=-\mathbf{i} P_{j}, j=1,2,3 .
\end{aligned}
$$

Operators $P_{\mu}$ are then generators of the $U(1) \times S U(2)$ Lie group of electro-weak interactions. We shall use

$$
\begin{aligned}
& \Psi_{L}=P_{+}(\Psi)=\left(\begin{array}{cc}
\phi_{L} & \phi_{n} \\
\hat{\phi}_{n} & \hat{\phi}_{L}
\end{array}\right) \\
& \Psi_{R}=P_{-}(\Psi)=\left(\begin{array}{cc}
\phi_{R} & 0 \\
0 & \hat{\phi}_{R}
\end{array}\right) ; \Psi=\Psi_{R}+\Psi_{L} \\
& P_{0}(\Psi)=\left(\Psi_{L}+2 \Psi_{R}\right) \gamma_{21}
\end{aligned}
$$

With $\gamma_{21}=\gamma_{2} \gamma_{1}$ and $\mathbf{i}=\gamma_{0123}=\gamma_{0} \gamma_{1} \gamma_{2} \gamma_{3}$. The last equality (2.5) comes from:

${ }^{5}$ We recall that $\left(D_{0}, D_{1}, D_{2}, D_{3}\right)$ is an orthogonal mobile basis in space-time, with $\left(D_{0}\right)^{2}=\rho_{e}^{2}$ and $\left(D_{j}\right)^{2}=-\rho_{e}^{2}, \quad j=1,2,3$. 


$$
\begin{aligned}
P_{0}(\Psi)=\Psi \gamma_{21}+P_{-}(\Psi) \mathbf{i}=\Psi \gamma_{21}+\Psi_{R} \mathbf{i} \\
\Psi_{R} \mathbf{i}=\left(\begin{array}{cc}
\phi_{R} & 0 \\
0 & \hat{\phi}_{R}
\end{array}\right)\left(\begin{array}{cc}
i & 0 \\
0 & -i
\end{array}\right)=\left(\begin{array}{cc}
\phi_{R} i & 0 \\
0 & \hat{\phi}_{R}(-i)
\end{array}\right) \\
\quad=\left(\begin{array}{cc}
\phi_{R} i \sigma_{3} & 0 \\
0 & \hat{\phi}_{R} i \sigma_{3}
\end{array}\right)=\left(\begin{array}{cc}
\phi_{R} & 0 \\
0 & \hat{\phi}_{R}
\end{array}\right)\left(\begin{array}{cc}
\sigma_{12} & 0 \\
0 & \sigma_{12}
\end{array}\right)=\Psi_{R} \gamma_{21}
\end{aligned}
$$

which results from our choice [1] of Dirac matrices:

$$
\gamma_{0}=\gamma^{0}=\left(\begin{array}{cc}
0 & I \\
I & 0
\end{array}\right) ; \quad I=\left(\begin{array}{cc}
1 & 0 \\
0 & 1
\end{array}\right) ; \gamma^{j}=-\gamma_{j}=\left(\begin{array}{cc}
0 & -\sigma_{j} \\
\sigma_{j} & 0
\end{array}\right)
$$

and we get

$$
\begin{aligned}
& P_{0}(\Psi)=\left(\Psi_{R}+\Psi_{L}\right) \gamma_{21}+\Psi_{R} \gamma_{21}=\left(\Psi_{L}+2 \Psi_{R}\right) \gamma_{21} \\
& \Psi_{L}+2 \Psi_{R}=\left(\begin{array}{cc}
\phi_{L}+2 \phi_{R} & \phi_{n} \\
\hat{\phi}_{n} & \hat{\phi}_{L}+2 \hat{\phi}_{R}
\end{array}\right)
\end{aligned}
$$

With (2.2) we get

$$
\begin{aligned}
\mathbf{D} \Psi & =\partial \Psi+\frac{g_{1}}{2} \mathbf{B} P_{0}(\Psi)+\frac{g_{2}}{2} \mathbf{W}^{j} P_{j}(\Psi) \\
& =\partial \Psi+\frac{g_{1}}{2} \mathbf{B}\left(\Psi_{L}+2 \Psi_{R}\right) \gamma_{21}+\frac{g_{2}}{2}\left[\mathbf{W}^{1} \Psi_{L} \gamma_{3} \mathbf{i}+\mathbf{W}^{2} \Psi_{L} \gamma_{3}+\mathbf{W}^{3} \Psi_{L}(-\mathbf{i})\right] \\
\partial= & \gamma^{\mu} \partial_{\mu} ; \mathbf{B}=\gamma^{\mu} B_{\mu} ; \mathbf{W}^{j}=\gamma^{\mu} W_{\mu}^{j}
\end{aligned}
$$

which gives

$$
\begin{aligned}
\mathbf{D} \Psi \gamma_{012}= & \partial \Psi \gamma_{012}+\frac{g_{1}}{2} \mathbf{B}\left(\Psi_{L}+2 \Psi_{R}\right) \gamma_{0} \\
& -\frac{g_{2}}{2}\left[\mathbf{W}^{1} \Psi_{L}+\mathbf{W}^{2} \Psi_{L} \mathbf{i}+\mathbf{W}^{3} \Psi_{L} \gamma_{3}\right]
\end{aligned}
$$

Next we get, with $\nabla=\sigma^{\mu} \partial_{\mu}=\partial_{0}-\left(\sigma_{1} \partial_{1}+\sigma_{2} \partial_{2}+\sigma_{3} \partial_{3}\right)$

$$
\begin{aligned}
\partial \Psi \gamma_{012} & =\left(\begin{array}{ll}
0 & \nabla \\
\hat{\nabla} & 0
\end{array}\right)\left(\begin{array}{ll}
\phi_{e} & \phi_{n} \\
\hat{\phi}_{n} & \hat{\phi}_{e}
\end{array}\right)\left(\begin{array}{cc}
0 & -i \sigma_{3} \\
-i \sigma_{3} & 0
\end{array}\right) \\
& =\left(\begin{array}{cc}
-i \nabla\left(\hat{\phi}_{R}+\hat{\phi}_{L}\right) \sigma_{3} & -i \nabla \hat{\phi}_{n} \sigma_{3} \\
-i \hat{\nabla} \phi_{n} \sigma_{3} & -i \hat{\nabla}\left(\phi_{R}+\phi_{L}\right) \sigma_{3}
\end{array}\right) \\
& =-i\left(\begin{array}{cc}
\nabla\left(-\hat{\phi}_{R}+\hat{\phi}_{L}\right) & \nabla \hat{\phi}_{n} \\
-\hat{\nabla} \phi_{n} & \hat{\nabla}\left(\phi_{R}-\phi_{L}\right)
\end{array}\right)
\end{aligned}
$$

From (2.8) and (2.9) we get with $B=B_{0}-\left(B_{1} \sigma_{1}+B_{2} \sigma_{2}+B_{3} \sigma_{3}\right)$

$$
\begin{aligned}
& \mathbf{B}\left(\Psi_{L}+2 \Psi_{R}\right) \gamma_{0}=\left(\begin{array}{cc}
0 & B \\
\hat{B} & 0
\end{array}\right)\left(\begin{array}{cc}
\phi_{L}+2 \phi_{R} & \phi_{n} \\
\hat{\phi}_{n} & \hat{\phi}_{L}+2 \hat{\phi}_{R}
\end{array}\right)\left(\begin{array}{ll}
0 & I \\
I & 0
\end{array}\right)=\left(\begin{array}{cc}
0 & B \\
\hat{B} & 0
\end{array}\right)\left(\begin{array}{cc}
\phi_{n} & \phi_{e}+\phi_{R} \\
\hat{\phi}_{e}+\hat{\phi}_{R} & \hat{\phi}_{n}
\end{array}\right) \\
& \mathbf{B} P_{0}(\Psi) \gamma_{012}=\left(\begin{array}{cc}
B\left(\hat{\phi}_{e}+\hat{\phi}_{R}\right) & B \hat{\phi}_{n} \\
\hat{B} \phi_{n} & \hat{B}\left(\phi_{e}+\phi_{R}\right)
\end{array}\right) .
\end{aligned}
$$

Next we get, with $W^{j}=W_{0}^{j}-\left(W_{1}^{j} \sigma_{1}+W_{2}^{j} \sigma_{2}+W_{3}^{j} \sigma_{3}\right)$ 


$$
\begin{aligned}
& \mathbf{W}^{1} \Psi_{L}=\left(\begin{array}{cc}
0 & W^{1} \\
\hat{W}^{1} & 0
\end{array}\right)\left(\begin{array}{ll}
\phi_{L} & \phi_{n} \\
\hat{\phi}_{n} & \hat{\phi}_{L}
\end{array}\right)=\left(\begin{array}{cc}
W^{1} \hat{\phi}_{n} & W^{1} \hat{\phi}_{L} \\
\hat{W}^{1} \phi_{L} & \hat{W}^{1} \phi_{n}
\end{array}\right) \\
& \mathbf{W}^{2} \Psi_{L} \mathbf{i}=\left(\begin{array}{cc}
0 & W^{2} \\
\hat{W}^{2} & 0
\end{array}\right)\left(\begin{array}{cc}
\phi_{L} & \phi_{n} \\
\hat{\phi}_{n} & \hat{\phi}_{L}
\end{array}\right)\left(\begin{array}{cc}
i & 0 \\
0 & -i
\end{array}\right)=\left(\begin{array}{cc}
i W^{2} \hat{\phi}_{n} & -i W^{2} \hat{\phi}_{L} \\
i \hat{W}^{2} \phi_{L} & -i \hat{W}^{2} \phi_{n}
\end{array}\right) \\
& \mathbf{W}^{3} \Psi_{L} \gamma_{3}=\left(\begin{array}{cc}
0 & W^{3} \\
\hat{W}^{3} & 0
\end{array}\right)\left(\begin{array}{cc}
\phi_{L} & \phi_{n} \\
\hat{\phi}_{n} & \hat{\phi}_{L}
\end{array}\right)\left(\begin{array}{cc}
0 & \sigma_{3} \\
-\sigma_{3} & 0
\end{array}\right)=\left(\begin{array}{ll}
-W^{3} \hat{\phi}_{L} \sigma_{3} & W^{3} \hat{\phi}_{n} \sigma_{3} \\
-\hat{W}^{3} \phi_{n} \sigma_{3} & \hat{W}^{3} \phi_{L} \sigma_{3}
\end{array}\right)=\left(\begin{array}{cc}
-W^{3} \hat{\phi}_{L} & W^{3} \hat{\phi}_{n} \\
\hat{W}^{3} \phi_{n} & -\hat{W}^{3} \phi_{L}
\end{array}\right) .
\end{aligned}
$$

We then get

$$
\mathbf{W}^{1} \Psi_{L}+\mathbf{W}^{2} \Psi_{L} \mathbf{i}+\mathbf{W}^{3} \Psi_{L} \gamma_{3}=\left(\begin{array}{ll}
\left(W^{1}+i W^{2}\right) \hat{\phi}_{n}-W^{3} \hat{\phi}_{L} & \left(W^{1}-i W^{2}\right) \hat{\phi}_{L}+W^{3} \hat{\phi}_{n} \\
\left(\hat{W}^{1}+i \hat{W}^{2}\right) \phi_{L}+\hat{W}^{3} \phi_{n} & \left(\hat{W}^{1}-i \hat{W}^{2}\right) \phi_{n}-\hat{W}^{3} \phi_{L}
\end{array}\right) .
$$

We next get

$$
\begin{aligned}
\tilde{\Psi} \mathbf{D} \Psi \gamma_{012} & =\tilde{\Psi} \partial \Psi \gamma_{012}+\frac{g_{1}}{2} \tilde{\Psi} \mathbf{B}\left(\Psi_{L}+2 \Psi_{R}\right) \gamma_{0}-\frac{g_{2}}{2} \tilde{\Psi}\left[\mathbf{W}^{1} \Psi_{L}+\mathbf{W}^{2} \Psi_{L} \mathbf{i}+\mathbf{W}^{3} \Psi_{L} \gamma_{3}\right] \\
\tilde{\Psi} \partial \Psi \gamma_{012} & =-i\left(\begin{array}{ll}
\bar{\phi}_{e} & \phi_{n}^{\dagger} \\
\bar{\phi}_{n} & \phi_{e}^{\dagger}
\end{array}\right)\left(\begin{array}{cc}
\nabla\left(-\hat{\phi}_{R}+\hat{\phi}_{L}\right) & \nabla \hat{\phi}_{n} \\
-\hat{\nabla} \phi_{n} & \hat{\nabla}\left(\phi_{R}-\phi_{L}\right)
\end{array}\right) \\
& =-i\left(\begin{array}{ll}
\bar{\phi}_{e} \nabla\left(-\hat{\phi}_{R}+\hat{\phi}_{L}\right)-\phi_{n}^{\dagger} \hat{\nabla} \phi_{n} & \bar{\phi}_{e} \nabla \hat{\phi}_{n}+\phi_{n}^{\dagger} \hat{\nabla}\left(\phi_{R}-\phi_{L}\right) \\
\bar{\phi}_{n} \nabla\left(-\hat{\phi}_{R}+\hat{\phi}_{L}\right)-\phi_{e}^{\dagger} \hat{\nabla} \phi_{n} & \bar{\phi}_{n} \nabla \hat{\phi}_{n}+\phi_{e}^{\dagger} \hat{\nabla}\left(\phi_{R}-\phi_{L}\right)
\end{array}\right)
\end{aligned}
$$

With our matrix representation (2.7) of the space-time algebra, the real part of a multivector is the real part of the scalar part of the matrix. Therefore we get

$$
\mathfrak{R}\left(\tilde{\Psi} \partial \Psi \gamma_{012}\right)=\mathfrak{R}\left[i \bar{\phi}_{e} \nabla\left(\hat{\phi}_{R}-\hat{\phi}_{L}\right)+i \phi_{n}^{\dagger} \hat{\nabla} \phi_{n}\right] .
$$

Next we get

$$
\begin{aligned}
& \tilde{\Psi} \mathbf{B} P_{0}(\Psi) \gamma_{012}=\left(\begin{array}{ll}
\bar{\phi}_{e} & \phi_{n}^{\dagger} \\
\bar{\phi}_{n} & \phi_{e}^{\dagger}
\end{array}\right)\left(\begin{array}{cc}
B\left(\hat{\phi}_{R}+\hat{\phi}_{e}\right) & B \hat{\phi}_{n} \\
\hat{B} \phi_{n} & \hat{B}\left(\phi_{R}+\phi_{e}\right)
\end{array}\right) \\
& =\left(\begin{array}{ll}
\bar{\phi}_{e} B\left(\hat{\phi}_{R}+\hat{\phi}_{e}\right)+\phi_{n}^{\dagger} \hat{B} \phi_{n} & \bar{\phi}_{e} B \hat{\phi}_{n}+\phi_{n}^{\dagger} \hat{B}\left(\phi_{R}+\phi_{e}\right) \\
\bar{\phi}_{n} B\left(\hat{\phi}_{R}+\hat{\phi}_{e}\right)+\phi_{e}^{\dagger} \hat{B} \phi_{n} & \bar{\phi}_{n} B \hat{\phi}_{n}+\phi_{e}^{\dagger} \hat{B}\left(\phi_{R}+\phi_{e}\right)
\end{array}\right) \\
& \mathfrak{R}\left[\tilde{\Psi} \mathbf{B} P_{0}(\Psi) \gamma_{012}\right]=\mathfrak{R}\left[\bar{\phi}_{e} B\left(\hat{\phi}_{R}+\hat{\phi}_{e}\right)+\phi_{n}^{\dagger} \hat{B} \phi_{n}\right] .
\end{aligned}
$$

From (2.10) and (2.14) we get

$$
\begin{gathered}
\tilde{\Psi}\left[\mathbf{W}^{1} \Psi_{L}+\mathbf{W}^{2} \Psi_{L} \mathbf{i}+\mathbf{W}^{3} \Psi_{L} \gamma_{3}\right] \\
=\left(\begin{array}{ll}
\bar{\phi}_{e} & \phi_{n}^{\dagger} \\
\bar{\phi}_{n} & \phi_{e}^{\dagger}
\end{array}\right)\left(\begin{array}{ll}
\left(W^{1}+i W^{2}\right) \hat{\phi}_{n}-W^{3} \hat{\phi}_{L} & \left(W^{1}-i W^{2}\right) \hat{\phi}_{L}+W^{3} \hat{\phi}_{n} \\
\left(\hat{W}^{1}+i \hat{W}^{2}\right) \phi_{L}+\hat{W}^{3} \phi_{n} & \left(\hat{W}^{1}-i \hat{W}^{2}\right) \phi_{n}-\hat{W}^{3} \phi_{L}
\end{array}\right)=\left(\begin{array}{ll}
U & V \\
\hat{V} & \hat{U}
\end{array}\right) \\
U=\bar{\phi}_{e}\left[\left(W^{1}+i W^{2}\right) \hat{\phi}_{n}-W^{3} \hat{\phi}_{L}\right]+\phi_{n}^{\dagger}\left[\left(\hat{W}^{1}+i \hat{W}^{2}\right) \phi_{L}+\hat{W}^{3} \phi_{n}\right]
\end{gathered}
$$

which gives

$$
\mathfrak{R}\left[\tilde{\Psi}\left[\mathbf{W}^{1} \Psi_{L}+\mathbf{W}^{2} \Psi_{L} \mathbf{i}+\mathbf{W}^{3} \Psi_{L} \gamma_{3}\right]\right]
$$




$$
=\Re\left(\bar{\phi}_{e} W^{1} \hat{\phi}_{n}+\phi_{n}^{\dagger} \hat{W}^{1} \phi_{L}\right)+\Re\left(i \bar{\phi}_{e} W^{2} \hat{\phi}_{n}+i \phi_{n}^{\dagger} \hat{W}^{2} \phi_{L}\right)+\Re\left(-\bar{\phi}_{e} W^{3} \hat{\phi}_{L}+\phi_{n}^{\dagger} \hat{W}^{3} \phi_{n}\right)
$$

We next get

$$
\begin{aligned}
2 \Re[ & {\left[i \bar{\phi}_{e} \nabla\left(\hat{\phi}_{R}-\hat{\phi}_{L}\right)+i \phi_{n}^{\dagger} \hat{\nabla} \phi_{n}\right] } \\
= & -i \eta_{1}^{*} \partial_{0} \eta_{1}+i \eta_{1} \partial_{0} \eta_{1}^{*}-i \eta_{2}^{*} \partial_{0} \eta_{2}+i \eta_{2} \partial_{0} \eta_{2}^{*}+i \xi_{2} \partial_{0} \xi_{2}^{*}-i \xi_{2}^{*} \partial_{0} \xi_{2}+i \xi_{1} \partial_{0} \xi_{1}^{*}-i \xi_{1}^{*} \partial_{0} \xi_{1}+i \zeta_{2} \partial_{0} \zeta_{2}^{*}-i \zeta_{2}^{*} \partial_{0} \zeta_{2} \\
& +i \zeta_{1} \partial_{0} \zeta_{1}^{*}-i \zeta_{1}^{*} \partial_{0} \zeta_{1}+i \eta_{1}^{*} \partial_{1} \eta_{2}-i \eta_{1} \partial_{1} \eta_{2}^{*}+i \eta_{2}^{*} \partial_{1} \eta_{1}-i \eta_{2} \partial_{1} \eta_{1}^{*}+i \xi_{2} \partial_{1} \xi_{1}^{*}-i \xi_{2}^{*} \partial_{1} \xi_{1}+i \xi_{1} \partial_{1} \xi_{2}^{*}-i \xi_{1}^{*} \partial_{1} \xi_{2} \\
& -i \zeta_{2} \partial_{1} \zeta_{1}^{*}+i \zeta_{2}^{*} \partial_{1} \zeta_{1}-i \zeta_{1} \partial_{1} \zeta_{2}^{*}+i \zeta_{1}^{*} \partial_{1} \zeta_{2}+\eta_{1}^{*} \partial_{2} \eta_{2}+\eta_{1} \partial_{2} \eta_{2}^{*}-\eta_{2}^{*} \partial_{2} \eta_{1}-\eta_{2} \partial_{2} \eta_{1}^{*}+\xi_{2} \partial_{2} \xi_{1}^{*}+\xi_{2}^{*} \partial_{2} \xi_{1} \\
& -\xi_{1} \partial_{2} \xi_{2}^{*}-\xi_{1}^{*} \partial_{2} \xi_{2}-\zeta_{2} \partial_{2} \zeta_{1}^{*}-\zeta_{2}^{*} \partial_{2} \zeta_{1}+\zeta_{1} \partial_{2} \zeta_{2}^{*}+\zeta_{1}^{*} \partial_{2} \zeta_{2}+i \eta_{1}^{*} \partial_{3} \eta_{1}-i \eta_{1} \partial_{3} \eta_{1}^{*}-i \eta_{2}^{*} \partial_{3} \eta_{2}+i \eta_{2} \partial_{3} \eta_{2}^{*} \\
& -i \xi_{2} \partial_{3} \xi_{2}^{*}+i \xi_{2}^{*} \partial_{3} \xi_{2}+i \xi_{1} \partial_{3} \xi_{1}^{*}-i \xi_{1}^{*} \partial_{3} \xi_{1}+i \zeta_{2} \partial_{3} \zeta_{2}^{*}-i \zeta_{2}^{*} \partial_{3} \zeta_{2}-i \zeta_{1} \partial_{3} \zeta_{1}^{*}+i \zeta_{1}^{*} \partial_{3} \zeta_{1} .
\end{aligned}
$$

For (2.17) we have

$$
\begin{aligned}
\Re & {\left[\bar{\phi}_{e} B\left(\hat{\phi}_{R}+\hat{\phi}_{e}\right)+\phi_{n}^{\dagger} \hat{B} \phi_{n}\right] } \\
= & B_{0}\left(\eta_{1} \eta_{1}^{*}+\eta_{2} \eta_{2}^{*}+2 \xi_{1} \xi_{1}^{*}+2 \xi_{2} \xi_{2}^{*}+\zeta_{1} \zeta_{1}^{*}+\zeta_{2} \zeta_{2}^{*}\right)+B_{1}\left(-\eta_{1} \eta_{2}^{*}-\eta_{2} \eta_{1}^{*}+2 \xi_{1} \xi_{2}^{*}+2 \xi_{2} \xi_{1}^{*}-\zeta_{1} \zeta_{2}^{*}-\zeta_{2} \zeta_{1}^{*}\right) \\
& +B_{2} i\left(-\eta_{1} \eta_{2}^{*}+\eta_{2} \eta_{1}^{*}+2 \xi_{1} \xi_{2}^{*}-2 \xi_{2} \xi_{1}^{*}-\zeta_{1} \zeta_{2}^{*}+\zeta_{2} \zeta_{1}^{*}\right)+B_{3}\left(-\eta_{1} \eta_{1}^{*}+\eta_{2} \eta_{2}^{*}+2 \xi_{1} \xi_{1}^{*}-2 \xi_{2} \xi_{2}^{*}-\zeta_{1} \zeta_{1}^{*}+\zeta_{2} \zeta_{2}^{*}\right)
\end{aligned}
$$

And for (2.19) we have

$$
\begin{gathered}
\mathfrak{R}\left(\bar{\phi}_{e} W^{1} \hat{\phi}_{n}+\phi_{n}^{\dagger} \hat{W}^{1} \phi_{L}\right)=W_{0}^{1}\left(\eta_{1} \zeta_{1}^{*}+\eta_{2} \zeta_{2}^{*}+\zeta_{1} \eta_{1}^{*}+\zeta_{2} \eta_{2}^{*}\right)+W_{1}^{1}\left(-\eta_{1} \zeta_{2}^{*}-\eta_{2} \zeta_{1}^{*}-\zeta_{1} \eta_{2}^{*}-\zeta_{2} \eta_{1}^{*}\right) \\
+W_{2}^{1} i\left(-\eta_{1} \zeta_{2}^{*}+\eta_{2} \zeta_{1}^{*}-\zeta_{1} \eta_{2}^{*}+\zeta_{2} \eta_{1}^{*}\right)+W_{3}^{1}\left(-\eta_{1} \zeta_{1}^{*}+\eta_{2} \zeta_{2}^{*}-\zeta_{1} \eta_{1}^{*}+\zeta_{2} \eta_{2}^{*}\right) \\
\mathfrak{R}\left(i \bar{\phi}_{e} W^{2} \hat{\phi}_{n}+i \phi_{n}^{\dagger} \hat{W}^{2} \phi_{L}\right)=W_{0}^{2} i\left(-\eta_{1} \zeta_{1}^{*}-\eta_{2} \zeta_{2}^{*}+\zeta_{1} \eta_{1}^{*}+\zeta_{2} \eta_{2}^{*}\right)+W_{1}^{2} i\left(\eta_{1} \zeta_{2}^{*}+\eta_{2} \zeta_{1}^{*}-\zeta_{1} \eta_{2}^{*}-\zeta_{2} \eta_{1}^{*}\right) \\
+W_{2}^{2}\left(-\eta_{1} \zeta_{2}^{*}+\eta_{2} \zeta_{1}^{*}+\zeta_{1} \eta_{2}^{*}-\zeta_{2} \eta_{1}^{*}\right)+W_{3}^{2} i\left(-\eta_{1} \zeta_{1}^{*}+\eta_{2} \zeta_{2}^{*}-\zeta_{1} \eta_{1}^{*}+\zeta_{2} \eta_{2}^{*}\right) \\
\Re\left(-\bar{\phi}_{e} W^{3} \hat{\phi}_{L}+\phi_{n}^{\dagger} \hat{W}^{3} \phi_{n}\right)=W_{0}^{3}\left(-\eta_{1} \eta_{1}^{*}-\eta_{2} \eta_{2}^{*}+\zeta_{1} \zeta_{1}^{*}+\zeta_{2} \zeta_{2}^{*}\right)+W_{1}^{3}\left(+\eta_{1} \eta_{2}^{*}+\eta_{2} \eta_{1}^{*}-\zeta_{1} \zeta_{2}^{*}-\zeta_{2} \zeta_{1}^{*}\right) \\
+W_{2}^{3} i\left(+\eta_{1} \eta_{2}^{*}-\eta_{2} \eta_{1}^{*}-\zeta_{1} \zeta_{2}^{*}+\zeta_{2} \zeta_{1}^{*}\right)+W_{3}^{3}\left(\eta_{1} \eta_{1}^{*}-\eta_{2} \eta_{2}^{*}-\zeta_{1} \zeta_{1}^{*}+\zeta_{2} \zeta_{2}^{*}\right)
\end{gathered}
$$

Therefore the Lagrangian density is

$$
\begin{aligned}
& 2 \mathcal{L}=-i \eta_{1}^{*} \partial_{0} \eta_{1}+i \eta_{1} \partial_{0} \eta_{1}^{*}-i \eta_{2}^{*} \partial_{0} \eta_{2}+i \eta_{2} \partial_{0} \eta_{2}^{*}+i \xi_{2} \partial_{0} \xi_{2}^{*}-i \xi_{2}^{*} \partial_{0} \xi_{2}+i \xi_{1} \partial_{0} \xi_{1}^{*}-i \xi_{1}^{*} \partial_{0} \xi_{1}+i \zeta_{2} \partial_{0} \zeta_{2}^{*}-i \zeta_{2}^{*} \partial_{0} \zeta_{2} \\
& +i \zeta_{1} \partial_{0} \zeta_{1}^{*}-i \zeta_{1}^{*} \partial_{0} \zeta_{1}+i \eta_{1}^{*} \partial_{1} \eta_{2}-i \eta_{1} \partial_{1} \eta_{2}^{*}+i \eta_{2}^{*} \partial_{1} \eta_{1}-i \eta_{2} \partial_{1} \eta_{1}^{*}+i \xi_{2} \partial_{1} \xi_{1}^{*}-i \xi_{2}^{*} \partial_{1} \xi_{1}+i \xi_{1} \partial_{1} \xi_{2}^{*}-i \xi_{1}^{*} \partial_{1} \xi_{2} \\
& -i \zeta_{2} \partial_{1} \zeta_{1}^{*}+i \zeta_{2}^{*} \partial_{1} \zeta_{1}-i \zeta_{1} \partial_{1} \zeta_{2}^{*}+i \zeta_{1}^{*} \partial_{1} \zeta_{2}+\eta_{1}^{*} \partial_{2} \eta_{2}+\eta_{1} \partial_{2} \eta_{2}^{*}-\eta_{2}^{*} \partial_{2} \eta_{1}-\eta_{2} \partial_{2} \eta_{1}^{*}+\xi_{2} \partial_{2} \xi_{1}^{*}+\xi_{2}^{*} \partial_{2} \xi_{1} \\
& -\xi_{1} \partial_{2} \xi_{2}^{*}-\xi_{1}^{*} \partial_{2} \xi_{2}-\zeta_{2} \partial_{2} \zeta_{1}^{*}-\zeta_{2}^{*} \partial_{2} \zeta_{1}+\zeta_{1} \partial_{2} \zeta_{2}^{*}+\zeta_{1}^{*} \partial_{2} \zeta_{2}+i \eta_{1}^{*} \partial_{3} \eta_{1}-i \eta_{1} \partial_{3} \eta_{1}^{*}-i \eta_{2}^{*} \partial_{3} \eta_{2}+i \eta_{2} \partial_{3} \eta_{2}^{*} \\
& -i \xi_{2} \partial_{3} \xi_{2}^{*}+i \xi_{2}^{*} \partial_{3} \xi_{2}+i \xi_{1} \partial_{3} \xi_{1}^{*}-i \xi_{1}^{*} \partial_{3} \xi_{1}+i \zeta_{2} \partial_{3} \zeta_{2}^{*}-i \zeta_{2}^{*} \partial_{3} \zeta_{2}-i \zeta_{1} \partial_{3} \zeta_{1}^{*}+i \zeta_{1}^{*} \partial_{3} \zeta_{1} \\
& +g_{1}\left[B_{0}\left(\eta_{1} \eta_{1}^{*}+\eta_{2} \eta_{2}^{*}+2 \xi_{1} \xi_{1}^{*}+2 \xi_{2} \xi_{2}^{*}+\zeta_{1} \zeta_{1}^{*}+\zeta_{2} \zeta_{2}^{*}\right)+B_{1}\left(-\eta_{1} \eta_{2}^{*}-\eta_{2} \eta_{1}^{*}+2 \xi_{1} \xi_{2}^{*}+2 \xi_{2} \xi_{1}^{*}-\zeta_{1} \zeta_{2}^{*}-\zeta_{2} \zeta_{1}^{*}\right)\right. \\
& \left.+B_{2} i\left(-\eta_{1} \eta_{2}^{*}+\eta_{2} \eta_{1}^{*}+2 \xi_{1} \xi_{2}^{*}-2 \xi_{2} \xi_{1}^{*}-\zeta_{1} \zeta_{2}^{*}+\zeta_{2} \zeta_{1}^{*}\right)+B_{3}\left(-\eta_{1} \eta_{1}^{*}+\eta_{2} \eta_{2}^{*}+2 \xi_{1} \xi_{1}^{*}-2 \xi_{2} \xi_{2}^{*}-\zeta_{1} \zeta_{1}^{*}+\zeta_{2} \zeta_{2}^{*}\right)\right] \\
& -g_{2}\left[W_{0}^{1}\left(\eta_{1} \zeta_{1}^{*}+\eta_{2} \zeta_{2}^{*}+\zeta_{1} \eta_{1}^{*}+\zeta_{2} \eta_{2}^{*}\right)+W_{0}^{2} i\left(-\eta_{1} \zeta_{1}^{*}-\eta_{2} \zeta_{2}^{*}+\zeta_{1} \eta_{1}^{*}+\zeta_{2} \eta_{2}^{*}\right)+W_{0}^{3}\left(-\eta_{1} \eta_{1}^{*}-\eta_{2} \eta_{2}^{*}+\zeta_{1} \zeta_{1}^{*}+\zeta_{2} \zeta_{2}^{*}\right)\right. \\
& +W_{1}^{1}\left(-\eta_{1} \zeta_{2}^{*}-\eta_{2} \zeta_{1}^{*}-\zeta_{1} \eta_{2}^{*}-\zeta_{2} \eta_{1}^{*}\right)+W_{1}^{2} i\left(\eta_{1} \zeta_{2}^{*}+\eta_{2} \zeta_{1}^{*}-\zeta_{1} \eta_{2}^{*}-\zeta_{2} \eta_{1}^{*}\right)+W_{1}^{3}\left(\eta_{1} \eta_{2}^{*}+\eta_{2} \eta_{1}^{*}-\zeta_{1} \zeta_{2}^{*}-\zeta_{2} \zeta_{1}^{*}\right) \\
& +W_{2}^{1} i\left(-\eta_{1} \zeta_{2}^{*}+\eta_{2} \zeta_{1}^{*}-\zeta_{1} \eta_{2}^{*}+\zeta_{2} \eta_{1}^{*}\right)+W_{2}^{2}\left(-\eta_{1} \zeta_{2}^{*}+\eta_{2} \zeta_{1}^{*}+\zeta_{1} \eta_{2}^{*}-\zeta_{2} \eta_{1}^{*}\right)+W_{2}^{3} i\left(\eta_{1} \eta_{2}^{*}-\eta_{2} \eta_{1}^{*}-\zeta_{1} \zeta_{2}^{*}+\zeta_{2} \zeta_{1}^{*}\right) \\
& \left.+W_{3}^{1}\left(-\eta_{1} \zeta_{1}^{*}+\eta_{2} \zeta_{2}^{*}-\zeta_{1} \eta_{1}^{*}+\zeta_{2} \eta_{2}^{*}\right)+W_{3}^{2} i\left(-\eta_{1} \zeta_{1}^{*}+\eta_{2} \zeta_{2}^{*}-\zeta_{1} \eta_{1}^{*}+\zeta_{2} \eta_{2}^{*}\right)+W_{3}^{3}\left(\eta_{1} \eta_{1}^{*}-\eta_{2} \eta_{2}^{*}-\zeta_{1} \zeta_{1}^{*}+\zeta_{2} \zeta_{2}^{*}\right)\right] \\
& +2 m \sqrt{a a^{*}+b b^{*}+c c^{*}}
\end{aligned}
$$


The Lagrange equation $\frac{\partial \mathcal{L}}{\partial \xi_{1}^{*}}=\partial_{\mu}\left(\frac{\partial \mathcal{L}}{\partial\left(\partial_{\mu} \xi_{1}^{*}\right)}\right)$ gives

$$
0=-i\left[\left(\partial_{0}+\partial_{3}\right) \xi_{1}+\left(\partial_{1}-i \partial_{2}\right) \xi_{2}\right]+g_{1}\left[\left(B_{0}+B_{3}\right) \xi_{1}+\left(B_{1}-i B_{2}\right) \xi_{2}\right]+\frac{m}{\rho}\left(a \eta_{1}+c \zeta_{1}\right)
$$

The Lagrange equation $\frac{\partial \mathcal{L}}{\partial \xi_{2}^{*}}=\partial_{\mu}\left(\frac{\partial \mathcal{L}}{\partial\left(\partial_{\mu} \xi_{2}^{*}\right)}\right)$ gives

$$
0=-i\left[\left(\partial_{1}+i \partial_{2}\right) \xi_{1}+\left(\partial_{0}-\partial_{3}\right) \xi_{2}\right]+g_{1}\left[\left(B_{1}+i B_{2}\right) \xi_{1}+\left(B_{0}-B_{3}\right) \xi_{2}\right]+\frac{m}{\rho}\left(a \eta_{2}+c \zeta_{2}\right)
$$

Together these equations read

$$
0=-i\left(\begin{array}{ll}
\partial_{0}+\partial_{3} & \partial_{1}-i \partial_{2} \\
\partial_{1}+i \partial_{2} & \partial_{0}-\partial_{3}
\end{array}\right)\left(\begin{array}{ll}
\xi_{1} & 0 \\
\xi_{2} & 0
\end{array}\right)+g_{1}\left(\begin{array}{cc}
B_{0}+B_{3} & B_{1}-i B_{2} \\
B_{1}+i B_{2} & B_{0}-B_{3}
\end{array}\right)\left(\begin{array}{ll}
\xi_{1} & 0 \\
\xi_{2} & 0
\end{array}\right)+\frac{m}{\rho}\left[a\left(\begin{array}{ll}
\eta_{1} & 0 \\
\eta_{2} & 0
\end{array}\right)+c\left(\begin{array}{ll}
\zeta_{1} & 0 \\
\zeta_{2} & 0
\end{array}\right)\right]
$$

Multiplying by $\sqrt{2}$ we get

$$
-i \hat{\nabla} \phi_{R}+g_{1} \hat{B} \phi_{R}+\frac{m}{\rho}\left(a \hat{\phi}_{L}+c \hat{\phi}_{n}\right)=0 .
$$

Since $\phi_{R} \sigma_{3}=\phi_{R}$ and $\hat{\phi}_{L} \sigma_{3}=\hat{\phi}_{L}$ this also reads

$$
\hat{\nabla} \phi_{R} \sigma_{21}+g_{1} \hat{B} \phi_{R}+\frac{m}{\rho}\left(a \hat{\phi}_{L}+c \hat{\phi}_{n}\right)=0
$$

then using the conjugation $M \mapsto \hat{M}$ we get

$$
\nabla \hat{\phi}_{R} \sigma_{21}+g_{1} B \hat{\phi}_{R}+\frac{m}{\rho}\left(a^{*} \phi_{L}+c^{*} \phi_{n}\right)=0
$$

The Lagrange equation $\frac{\partial \mathcal{L}}{\partial \eta_{1}^{*}}=\partial_{\mu}\left(\frac{\partial \mathcal{L}}{\partial\left(\partial_{\mu} \eta_{1}^{*}\right)}\right)$ gives

$$
\begin{gathered}
0=-i\left[\left(\partial_{0}-\partial_{3}\right) \eta_{1}+\left(-\partial_{1}+i \partial_{2}\right) \eta_{2}\right]+\frac{g_{1}}{2}\left[\left(B_{0}-B_{3}\right) \eta_{1}+\left(-B_{1}+i B_{2}\right) \eta_{2}\right] \\
-\frac{g_{2}}{2}\left(\begin{array}{c}
\left(W_{0}^{1}-W_{3}^{1}\right) \zeta_{1}+\left(-W_{1}^{1}+i W_{2}^{1}\right) \zeta_{2} \\
+i\left[\left(W_{0}^{2}-W_{3}^{2}\right) \zeta_{1}+\left(-W_{1}^{2}+i W_{2}^{2}\right) \zeta_{2}\right] \\
-\left(W_{0}^{3}-W_{3}^{3}\right) \eta_{1}-\left(-W_{1}^{3}+i W_{2}^{3}\right) \eta_{2}
\end{array}\right)+\frac{m}{\rho}\left(a^{*} \xi_{1}+b \zeta_{2}^{*}\right)
\end{gathered}
$$

The Lagrange equation $\frac{\partial \mathcal{L}}{\partial \eta_{2}^{*}}=\partial_{\mu}\left(\frac{\partial \mathcal{L}}{\partial\left(\partial_{\mu} \eta_{2}^{*}\right)}\right)$ gives

$$
\begin{array}{r}
0=-i\left[\left(-\partial_{1}-i \partial_{2}\right) \eta_{1}+\left(\partial_{0}+\partial_{3}\right) \eta_{2}\right]+\frac{g_{1}}{2}\left[\left(-B_{1}-i B_{2}\right) \eta_{1}+\left(B_{0}+B_{3}\right) \eta_{2}\right] \\
-\frac{g_{2}}{2}\left(\begin{array}{c}
\left(-W_{1}^{1}-i W_{2}^{1}\right) \zeta_{1}+\left(W_{0}^{1}+W_{3}^{1}\right) \zeta_{2} \\
+i\left[\left(-W_{1}^{2}-i W_{2}^{2}\right) \zeta_{1}+\left(W_{0}^{2}+W_{3}^{2}\right) \zeta_{2}\right] \\
-\left(-W_{1}^{3}-i W_{2}^{3}\right) \eta_{1}-\left(W_{0}^{3}+W_{3}^{3}\right) \eta_{2}
\end{array}\right)+\frac{m}{\rho}\left(a^{*} \xi_{2}-b \zeta_{1}^{*}\right)
\end{array}
$$

Together these equations read 


$$
\begin{aligned}
0= & -i\left(\begin{array}{cc}
\partial_{0}-\partial_{3} & -\partial_{1}+i \partial_{2} \\
-\partial_{1}-i \partial_{2} & \partial_{0}+\partial_{3}
\end{array}\right)\left(\begin{array}{ll}
\eta_{1} & 0 \\
\eta_{2} & 0
\end{array}\right)+\frac{g_{1}}{2}\left(\begin{array}{cc}
B_{0}-B_{3} & -B_{1}+i B_{2} \\
-B_{1}-i B_{2} & B_{0}+B_{3}
\end{array}\right)\left(\begin{array}{cc}
\eta_{1} & 0 \\
\eta_{2} & 0
\end{array}\right) \\
& -\frac{g_{2}}{2}\left[\left(\begin{array}{cc}
W_{0}^{1}-W_{3}^{1} & -W_{1}^{1}+i W_{2}^{1} \\
-W_{1}^{1}-i W_{2}^{1} & W_{0}^{1}+W_{3}^{1}
\end{array}\right)\left(\begin{array}{ll}
\zeta_{1} & 0 \\
\zeta_{2} & 0
\end{array}\right)+i\left(\begin{array}{cc}
W_{0}^{2}-W_{3}^{2} & -W_{1}^{2}+i W_{2}^{2} \\
-W_{1}^{2}-i W_{2}^{2} & W_{0}^{2}+W_{3}^{2}
\end{array}\right)\left(\begin{array}{ll}
\zeta_{1} & 0 \\
\zeta_{2} & 0
\end{array}\right)\right. \\
& \left.-\left(\begin{array}{cc}
W_{0}^{3}-W_{3}^{3} & -W_{1}^{3}+i W_{2}^{3} \\
-W_{1}^{3}-i W_{2}^{3} & W_{0}^{3}+W_{3}^{3}
\end{array}\right)\left(\begin{array}{ll}
\eta_{1} & 0 \\
\eta_{2} & 0
\end{array}\right)\right]+\frac{m}{\rho}\left[a^{*}\left(\begin{array}{cc}
\xi_{1} & 0 \\
\xi_{2} & 0
\end{array}\right)+b\left(\begin{array}{cc}
\zeta_{2}^{*} & 0 \\
-\zeta_{1}^{*} & 0
\end{array}\right)\right]
\end{aligned}
$$

Multiplying by $\sqrt{2}$ this reads

$$
0=\nabla \hat{\phi}_{L} \sigma_{21}+\frac{g_{1}}{2} B \hat{\phi}_{L}+\frac{g_{2}}{2}\left[-\left(W^{1}+i W^{2}\right) \hat{\phi}_{n}+W^{3} \hat{\phi}_{L}\right]+\frac{m}{\rho}\left(a^{*} \phi_{R}-b \phi_{n} \sigma_{1}\right)
$$

Adding (2.31) and (2.35) we get the wave equation

$$
0=\nabla \hat{\phi}_{e} \sigma_{21}+\frac{g_{1}}{2} B\left(\hat{\phi}_{L}+2 \hat{\phi}_{R}\right)+\frac{g_{2}}{2}\left[-\left(W^{1}+i W^{2}\right) \hat{\phi}_{n}+W^{3} \hat{\phi}_{L}\right]+\frac{m}{\rho}\left(a^{*} \phi_{e}-b \phi_{n} \sigma_{1}+c^{*} \phi_{n}\right)
$$

Without its mass term, this equation is the wave equation of the electron in the electro-weak theory [1] (6.57). The great difference is that there is now a mass term, and since the Lagrangian density is both relativistic and gauge invariant, we shall see that the wave equation with mass term conserves these invariant properties. The Lagrange equation $\frac{\partial \mathcal{L}}{\partial \zeta_{1}^{*}}=\partial_{\mu}\left(\frac{\partial \mathcal{L}}{\partial\left(\partial_{\mu} \zeta_{1}^{*}\right)}\right)$ gives

$$
\begin{aligned}
0= & -i\left[\left(\partial_{0}-\partial_{3}\right) \zeta_{1}+\left(-\partial_{1}+i \partial_{2}\right) \zeta_{2}\right]+\frac{g_{1}}{2}\left[\left(B_{0}-B_{3}\right) \zeta_{1}+\left(-B_{1}+i B_{2}\right) \zeta_{2}\right] \\
+ & +\frac{g_{2}}{2}\left(\begin{array}{c}
- \\
-\left[\left(W_{0}^{1}-W_{3}^{1}\right) \eta_{1}+\left(-W_{1}^{1}+i W_{2}^{1}\right) \eta_{2}\right] \\
+i\left[\left(W_{0}^{2}-W_{3}^{2}\right) \eta_{1}+\left(-W_{1}^{2}+i W_{2}^{2}\right) \eta_{2}\right] \\
-\left(W_{0}^{3}-W_{3}^{3}\right) \zeta_{1}-\left(-W_{1}^{3}+i W_{2}^{3}\right) \zeta_{2}
\end{array}\right)+\frac{m}{\rho}\left(c^{*} \xi_{1}-b \eta_{2}^{*}\right)
\end{aligned}
$$

The Lagrange equation $\frac{\partial \mathcal{L}}{\partial \zeta_{2}^{*}}=\partial_{\mu}\left(\frac{\partial \mathcal{L}}{\partial\left(\partial_{\mu} \zeta_{2}^{*}\right)}\right)$ gives

$$
\begin{aligned}
0= & -i\left[\left(-\partial_{1}-i \partial_{2}\right) \zeta_{1}+\left(\partial_{0}+\partial_{3}\right) \zeta_{2}\right]+\frac{g_{1}}{2}\left[\left(-B_{1}-i B_{2}\right) \zeta_{1}+\left(B_{0}+B_{3}\right) \zeta_{2}\right] \\
+ & \frac{g_{2}}{2}\left(\begin{array}{c}
- \\
-\left[\left(-W_{1}^{1}-i W_{2}^{1}\right) \eta_{1}+\left(W_{0}^{1}+W_{3}^{1}\right) \eta_{2}\right] \\
+i\left[\left(-W_{1}^{2}-i W_{2}^{2}\right) \eta_{1}+\left(W_{0}^{2}+W_{3}^{2}\right) \eta_{2}\right] \\
-\left(-W_{1}^{3}-i W_{2}^{3}\right) \zeta_{1}-\left(W_{0}^{3}+W_{3}^{3}\right) \zeta_{2}
\end{array}\right)+\frac{m}{\rho}\left(c^{*} \xi_{2}+b \eta_{1}^{*}\right)
\end{aligned}
$$

Together these equations read

$$
\begin{aligned}
0= & -i\left(\begin{array}{cc}
\partial_{0}-\partial_{3} & -\partial_{1}+i \partial_{2} \\
-\partial_{1}-i \partial_{2} & \partial_{0}+\partial_{3}
\end{array}\right)\left(\begin{array}{cc}
\zeta_{1} & 0 \\
\zeta_{2} & 0
\end{array}\right)+\frac{g_{1}}{2}\left(\begin{array}{cc}
B_{0}-B_{3} & -B_{1}+i B_{2} \\
-B_{1}-i B_{2} & B_{0}+B_{3}
\end{array}\right)\left(\begin{array}{cc}
\zeta_{1} & 0 \\
\zeta_{2} & 0
\end{array}\right) \\
& +\frac{g_{2}}{2}\left[-\left(\begin{array}{cc}
W_{0}^{1}-W_{3}^{1} & -W_{1}^{1}+i W_{2}^{1} \\
-W_{1}^{1}-i W_{2}^{1} & W_{0}^{1}+W_{3}^{1}
\end{array}\right)\left(\begin{array}{ll}
\eta_{1} & 0 \\
\eta_{2} & 0
\end{array}\right)+i\left(\begin{array}{cc}
W_{0}^{2}-W_{3}^{2} & -W_{1}^{2}+i W_{2}^{2} \\
-W_{1}^{2}-i W_{2}^{2} & W_{0}^{2}+W_{3}^{2}
\end{array}\right)\left(\begin{array}{ll}
\eta_{1} & 0 \\
\eta_{2} & 0
\end{array}\right)\right. \\
& \left.-\left(\begin{array}{cc}
W_{0}^{3}-W_{3}^{3} & -W_{1}^{3}+i W_{2}^{3} \\
-W_{1}^{3}-i W_{2}^{3} & W_{0}^{3}+W_{3}^{3}
\end{array}\right)\left(\begin{array}{ll}
\zeta_{1} & 0 \\
\zeta_{2} & 0
\end{array}\right)\right]+\frac{m}{\rho}\left[b\left(\begin{array}{cc}
-\eta_{2}^{*} & 0 \\
\eta_{1}^{*} & 0
\end{array}\right)+c^{*}\left(\begin{array}{cc}
\xi_{1} & 0 \\
\xi_{2} & 0
\end{array}\right)\right]
\end{aligned}
$$


Multiplying by $\sqrt{2}$ this reads

$$
0=\nabla \hat{\phi}_{n} \sigma_{21}+\frac{g_{1}}{2} B \hat{\phi}_{n}+\frac{g_{2}}{2}\left[\left(-W^{1}+i W^{2}\right) \hat{\phi}_{L}-W^{3} \hat{\phi}_{n}\right]+\frac{m}{\rho}\left(c^{*} \phi_{R}+b \phi_{L} \sigma_{1}\right)
$$

Without the mass term, this equation is the wave equation of the electronic neutrino in the electro-weak theory [1] (6.58). Following backwards the calculation from (2.10) to (2.15) we can see that the system (2.36) (2.40) is equivalent to the wave equation

$$
\mathbf{D} \Psi \gamma_{012}+m \rho \chi=0
$$

where

$$
\chi=\frac{1}{\rho^{2}}\left(\begin{array}{cc}
a^{*} \phi_{e}-b \phi_{n} \sigma_{1}+c^{*} \phi_{n} & b \phi_{L} \sigma_{1}+c^{*} \phi_{R} \\
-b^{*} \hat{\phi}_{L} \sigma_{1}+c \hat{\phi}_{R} & a \hat{\phi}_{e}+b^{*} \hat{\phi}_{n} \sigma_{1}+c \hat{\phi}_{n}
\end{array}\right)
$$

or to the invariant equation

$$
\tilde{\Psi} \mathbf{D} \Psi \gamma_{012}+m \rho \tilde{\Psi} \chi=0
$$

Since this wave equation is not exactly our starting one, we must explain how this equation has exactly the Lagrangian equation $\mathcal{L}=0$ as real part. We shall next prove the relativistic invariance and the gauge invariance of this wave equation under the electro-weak gauge group.

\section{Invariances}

With (2.2) and (2.42) we get

$$
\begin{gathered}
\rho^{2} \tilde{\Psi} \chi=\left(\begin{array}{cc}
\bar{\phi}_{e} & \phi_{n}^{\dagger} \\
\bar{\phi}_{n} & \phi_{e}^{\dagger}
\end{array}\right)\left(\begin{array}{cc}
a^{*} \phi_{e}-b \phi_{n} \sigma_{1}+c^{*} \phi_{n} & b \phi_{L} \sigma_{1}+c^{*} \phi_{R} \\
-b^{*} \hat{\phi}_{L} \sigma_{1}+c \hat{\phi}_{R} & a \hat{\phi}_{e}+b^{*} \hat{\phi}_{n} \sigma_{1}+c \hat{\phi}_{n}
\end{array}\right)=\left(\begin{array}{cc}
U & V \\
\hat{V} & \hat{U}
\end{array}\right) \\
U=a^{*} \bar{\phi}_{e} \phi_{e}-b \bar{\phi}_{e} \phi_{n} \sigma_{1}+c^{*} \bar{\phi}_{e} \phi_{n}-b^{*} \phi_{n}^{\dagger} \hat{\phi}_{L} \sigma_{1}+c \phi_{n}^{\dagger} \hat{\phi}_{R} .
\end{gathered}
$$

We get

$$
\begin{gathered}
a^{*} \bar{\phi}_{e} \phi_{e}=a a^{*} ;-b \bar{\phi}_{e} \phi_{n} \sigma_{1}-b^{*} \phi_{n}^{\dagger} \hat{\phi}_{L} \sigma_{1}=\left(\begin{array}{cc}
b b^{*} & 0 \\
-b c & b b^{*}
\end{array}\right)=b b^{*}+\frac{b c}{2}\left(-\sigma_{1}+i \sigma_{2}\right) \\
c^{*} \bar{\phi}_{e} \phi_{n}+c \phi_{n}^{\dagger} \hat{\phi}_{R}=\left(\begin{array}{cc}
0 & -b^{*} c^{*} \\
0 & 2 c c^{*}
\end{array}\right)=c c^{*}\left(1-\sigma_{3}\right)+\frac{b^{*} c^{*}}{2}\left(-\sigma_{1}-i \sigma_{2}\right) \\
U=\rho^{2}-\Re(b c) \sigma_{1}-\Im(b c) \sigma_{2}-c c^{*} \sigma_{3} \\
\Re(m \rho \tilde{\Psi} \chi)=m \rho .
\end{gathered}
$$

Therefore the Lagrangian density (2.25) is also the real part of the invariant form (2.43) of the wave equation. The value of $V$ is more simple because we get

$$
V=a c^{*} \text {. }
$$

We now review the form invariance of this wave equation; next we shall prove its gauge invariance.

\subsection{Form Invariance}

Under the $C l_{3}^{*}=G l(2, \mathbb{C})$ multiplicative Lie group made of any invertible matrix $M$ satisfying (1.10) We got [1] [3]

$$
\begin{aligned}
& \nabla=\bar{M} \nabla^{\prime} \hat{M} ; \Psi^{\prime}=N \Psi \\
& \mathbf{D}=\tilde{N} \mathbf{D}^{\prime} N ; \quad N=\left(\begin{array}{cc}
M & 0 \\
0 & \hat{M}
\end{array}\right) ; \quad \tilde{N}=\left(\begin{array}{cc}
\bar{M} & 0 \\
0 & M^{\dagger}
\end{array}\right) \\
& 0=\tilde{\Psi} \mathbf{D} \Psi \gamma_{012}+m \rho \tilde{\Psi} \chi=\tilde{\Psi} \tilde{N} \mathbf{D}^{\prime} N \Psi \gamma_{012}+m \rho \tilde{\Psi} \chi=\tilde{\Psi}^{\prime} \mathbf{D}^{\prime} \Psi^{\prime} \gamma_{012}+m^{\prime} \rho^{\prime} \tilde{\Psi} \chi
\end{aligned}
$$


And we shall get the form invariance of the wave equation if and only if

$$
\begin{aligned}
& \tilde{\Psi} \chi=\tilde{\Psi}^{\prime} \chi^{\prime}=\tilde{\Psi} \tilde{N} \chi^{\prime} \\
& \chi^{\prime}=\tilde{N}^{-1} \chi
\end{aligned}
$$

From (1.13), (1.16), (1.18) we get with (1.11)

$$
\begin{aligned}
& \frac{1}{\rho^{\prime 2}}\left(a^{\prime *} \phi_{e}^{\prime}-b^{\prime} \phi_{n}^{\prime} \sigma_{1}+c^{\prime *} \phi_{n}^{\prime}\right)=\frac{1}{r^{2} \rho^{2}}\left(r \mathrm{e}^{-i \theta} a^{*} M \phi_{e}-r \mathrm{e}^{-i \theta} b M \phi_{n} \sigma_{1}+r \mathrm{e}^{-i \theta} c^{*} M \phi_{n}\right) \\
& =\frac{M}{r \mathrm{e}^{i \theta}} \frac{1}{\rho^{2}}\left(a^{*} \phi_{e}-b \phi_{n} \sigma_{1}+c^{*} \phi_{n}\right)=\bar{M}^{-1} \frac{1}{\rho^{2}}\left(a^{*} \phi_{e}-b \phi_{n} \sigma_{1}+c^{*} \phi_{n}\right) . \\
& \frac{1}{\rho^{\prime 2}}\left(b^{\prime} \phi_{L}^{\prime} \sigma_{1}+c^{\prime *} \phi_{n}^{\prime}\right)=\frac{1}{r^{2} \rho^{2}}\left(r \mathrm{e}^{-i \theta} b M \phi_{L} \sigma_{1}+r \mathrm{e}^{-i \theta} c^{*} M \phi_{n}\right) \\
& =\frac{M}{r \mathrm{e}^{i \theta}} \frac{1}{\rho^{2}}\left(b \phi_{L} \sigma_{1}+c^{*} \phi_{n}\right)=\bar{M}^{-1} \frac{1}{\rho^{2}}\left(b \phi_{L} \sigma_{1}+c^{*} \phi_{n}\right) .
\end{aligned}
$$

This gives (3.5) since

$$
\chi^{\prime}=\left(\begin{array}{cc}
\bar{M}^{-1} & 0 \\
0 & M^{\dagger-1}
\end{array}\right) \chi=\tilde{N}^{-1} \chi .
$$

And the wave equation is form invariant under $\mathrm{Cl}_{3}^{*}$ then it is relativistic invariant.

\subsection{Gauge Invariance-Group Generated by $P_{0}$}

We shall use a convenient form of the projector $P_{0}$ (proof in [1] B p.143)

$$
\begin{aligned}
& P_{0}(\Psi)=\frac{\mathbf{i}}{2} \Psi+\frac{3}{2} \Psi \gamma_{21} \\
& \Psi^{\prime}=\left[\exp \left(\theta P_{0}\right)\right](\Psi)=\mathrm{e}^{\frac{\theta}{2} \mathbf{i}} \Psi \mathrm{e}^{\frac{3 \theta}{2} \gamma_{21}}
\end{aligned}
$$

We have proved ([1] (B.14)) that

$$
\begin{aligned}
& \mathbf{D}^{\prime} \Psi^{\prime}=\mathrm{e}^{-\frac{\theta}{2} \mathbf{i}}(\mathbf{D} \Psi) \mathrm{e}^{\frac{3 \theta}{2} \gamma_{21}} \\
& \mathbf{D}^{\prime} \Psi^{\prime} \gamma_{012}=\mathrm{e}^{-\frac{\theta}{2}}\left(\mathbf{D} \Psi \gamma_{012}\right) \mathrm{e}^{\frac{3 \theta}{2} \gamma_{21}}
\end{aligned}
$$

Equation (3.8) reads

$$
\begin{aligned}
\left(\begin{array}{cc}
\phi_{e}^{\prime} & \phi_{n}^{\prime} \\
\hat{\phi}_{n}^{\prime} & \hat{\phi}_{e}^{\prime}
\end{array}\right) & =\left(\begin{array}{cc}
\mathrm{e}^{i \frac{\theta}{2}} & 0 \\
0 & \mathrm{e}^{-i \frac{\theta}{2}}
\end{array}\right)\left(\begin{array}{cc}
\phi_{e} & \phi_{n} \\
\hat{\phi}_{n} & \hat{\phi}_{e}
\end{array}\right)\left(\begin{array}{cc}
\mathrm{e}^{\frac{3 \theta}{2} i \sigma_{3}} & 0 \\
0 & \mathrm{e}^{\frac{3 \theta}{2} i \sigma_{3}}
\end{array}\right) \\
& =\left(\begin{array}{cc}
\mathrm{e}^{i \frac{\theta}{2}} \phi_{e} \mathrm{e}^{\frac{3 \theta}{2} i \sigma_{3}} & \mathrm{e}^{i \frac{\theta}{2}} \phi_{n} \mathrm{e}^{\frac{3 \theta}{2} i \sigma_{3}} \\
\mathrm{e}^{-i \frac{\theta}{2}} \hat{\phi}_{n} \mathrm{e}^{\frac{3 \theta}{2} i \sigma_{3}} & \mathrm{e}^{-i \frac{\theta}{2}} \hat{\phi}_{e} \mathrm{e}^{\frac{3 \theta}{2} i \sigma_{3}}
\end{array}\right)
\end{aligned}
$$

This gives

$$
\begin{aligned}
& \left(\begin{array}{cc}
\xi_{1}^{\prime} & -\eta_{2}^{\prime *} \\
\xi_{2}^{\prime} & \eta_{1}^{\prime *}
\end{array}\right)=\left(\begin{array}{cc}
\mathrm{e}^{2 i \theta} \xi_{1} & -\mathrm{e}^{-i \theta} \eta_{2}^{*} \\
\mathrm{e}^{2 i \theta} \xi_{2} & \mathrm{e}^{-i \theta} \eta_{1}^{*}
\end{array}\right) ; \\
& \left(\begin{array}{cc}
0 & -\zeta_{2}^{\prime *} \\
0 & \zeta_{1}^{\prime *}
\end{array}\right)=\left(\begin{array}{cc}
0 & -\mathrm{e}^{-i \theta} \zeta_{2}^{*} \\
0 & \mathrm{e}^{-i \theta} \zeta_{1}^{*}
\end{array}\right)
\end{aligned}
$$

And we get 


$$
\left(\begin{array}{l}
\xi_{1}^{\prime} \\
\xi_{2}^{\prime}
\end{array}\right)=\mathrm{e}^{2 i \theta}\left(\begin{array}{l}
\xi_{1} \\
\xi_{2}
\end{array}\right) ;\left(\begin{array}{l}
\eta_{1}^{\prime} \\
\eta_{2}^{\prime}
\end{array}\right)=\mathrm{e}^{\mathrm{i} \theta}\left(\begin{array}{l}
\eta_{1} \\
\eta_{2}
\end{array}\right) ;\left(\begin{array}{l}
\zeta_{1}^{\prime} \\
\zeta_{2}^{\prime}
\end{array}\right)=\mathrm{e}^{\mathrm{i} \theta}\left(\begin{array}{l}
\zeta_{1} \\
\zeta_{2}
\end{array}\right) .
$$

We then get for $a, b, c$ :

$$
\begin{aligned}
& a^{\prime}=\mathrm{e}^{\mathrm{i} \theta} a ; \quad a^{\prime} a^{\prime^{*}}=a a^{*} \\
& b^{\prime}=\mathrm{e}^{2 i \theta} b ; \quad b^{\prime} b^{\prime^{*}}=b b^{*} \\
& c^{\prime}=\mathrm{e}^{i \theta} c ; \quad c^{\prime} c^{\prime *}=c c^{*} \\
& \rho^{\prime}=\rho
\end{aligned}
$$

We must study

$$
\chi^{\prime}=\frac{1}{\rho^{\prime 2}}\left(\begin{array}{cc}
a^{\prime *} \phi_{e}^{\prime}-b^{\prime} \phi_{n}^{\prime} \sigma_{1}+c^{\prime *} \phi_{n}^{\prime} & b^{\prime} \phi_{L}^{\prime} \sigma_{1}+c^{\prime *} \phi_{R}^{\prime} \\
-b^{\prime *} \hat{\phi}_{L}^{\prime} \sigma_{1}+c^{\prime} \hat{\phi}_{R}^{\prime} & a^{\prime} \hat{\phi}_{e}^{\prime}+b^{\prime *} \hat{\phi}_{n}^{\prime} \sigma_{1}+c^{\prime} \hat{\phi}_{n}^{\prime}
\end{array}\right)
$$

We get

$$
\begin{aligned}
a^{\prime *} \phi_{e}^{\prime}-b^{\prime} \phi_{n}^{\prime} \sigma_{1}+c^{\prime *} \phi_{n}^{\prime} & =\mathrm{e}^{-i \theta} a^{*} \mathrm{e}^{i \frac{\theta}{2}} \phi_{e} \mathrm{e}^{\frac{3 \theta}{2} i \sigma_{3}}-\mathrm{e}^{2 i \theta} b \mathrm{e}^{i \frac{\theta}{2}} \phi_{n} \mathrm{e}^{\frac{3 \theta}{2} i \sigma_{3}} \sigma_{1}+\mathrm{e}^{-i \theta} c^{*} \mathrm{e}^{i \frac{\theta}{2}} \phi_{n} \mathrm{e}^{\frac{3 \theta}{2} i \sigma_{3}} \\
& =\mathrm{e}^{-i \frac{\theta}{2}}\left(a^{*} \phi_{e}-\mathrm{e}^{3 i \theta} b \phi_{n} \mathrm{e}^{3 i \theta \sigma_{3}} \sigma_{1}+c^{*} \phi_{n}\right) \mathrm{e}^{\frac{3 \theta}{2} i \sigma_{3}} \\
& =\mathrm{e}^{-i \frac{\theta}{2}}\left(a^{*} \phi_{e}-\mathrm{e}^{3 i \theta} b \phi_{n} \mathrm{e}^{-3 i \theta} \sigma_{1}+c^{*} \phi_{n}\right) \mathrm{e}^{\frac{3 \theta}{2} i \sigma_{3}} \\
& =\mathrm{e}^{-i \frac{\theta}{2}}\left(a^{*} \phi_{e}-b \phi_{n} \sigma_{1}+c^{*} \phi_{n}\right) \mathrm{e}^{\frac{3 \theta}{2} i \sigma_{3}}
\end{aligned}
$$

and similarly

$$
\begin{aligned}
b^{\prime} \phi_{L}^{\prime} \sigma_{1}+c^{\prime *} \phi_{R}^{\prime} & =\mathrm{e}^{2 i \theta} b \mathrm{e}^{i \frac{\theta}{2}} \phi_{L} \mathrm{e}^{\frac{3 \theta}{2} i \sigma_{3}} \sigma_{1}+\mathrm{e}^{-i \theta} c^{*} \mathrm{e}^{i \frac{\theta}{2}} \phi_{R} \mathrm{e}^{\frac{3 \theta}{2} i \sigma_{3}} \\
& =\mathrm{e}^{i \frac{5 \theta}{2}} b \phi_{L} \mathrm{e}^{3 i \theta \sigma_{3}} \sigma_{1} \mathrm{e}^{\frac{3 \theta}{2} i \sigma_{3}}+\mathrm{e}^{-i \frac{\theta}{2}} c^{*} \phi_{R} \mathrm{e}^{\frac{3 \theta}{2} i \sigma_{3}} \\
& =\left(\mathrm{e}^{i \frac{5 \theta}{2}} b \phi_{L} \mathrm{e}^{-3 i \theta} \sigma_{1}+\mathrm{e}^{-i \frac{\theta}{2}} c^{*} \phi_{R}\right) \mathrm{e}^{\frac{3 \theta}{2} i \sigma_{3}} \\
& =\mathrm{e}^{-i \frac{\theta}{2}}\left(b \phi_{L} \sigma_{1}+c^{*} \phi_{R}\right) \mathrm{e}^{\frac{3 \theta}{2} i \sigma_{3}}
\end{aligned}
$$

This gives

$$
\chi^{\prime}=\frac{1}{\rho^{2}}\left(\begin{array}{cc}
\mathrm{e}^{-i \frac{\theta}{2}}\left(a^{*} \phi_{e}-b \phi_{n} \sigma_{1}+c^{*} \phi_{n}\right) \mathrm{e}^{\frac{3 \theta}{2} i \sigma_{3}} & \mathrm{e}^{-i \frac{\theta}{2}}\left(b \phi_{L} \sigma_{1}+c^{*} \phi_{R}\right) \mathrm{e}^{\frac{3 \theta}{2} i \sigma_{3}} \\
\mathrm{e}^{i \frac{\theta}{2}}\left(-b^{*} \hat{\phi}_{L} \sigma_{1}+c \hat{\phi}_{R}\right) \mathrm{e}^{\frac{3 \theta}{2} i \sigma_{3}} & \mathrm{e}^{i \frac{\theta}{2}}\left(a \hat{\phi}_{e}+b \hat{\phi}_{n} \sigma_{1}+c \hat{\phi}_{n}\right) \mathrm{e}^{\frac{3 \theta}{2} i \sigma_{3}}
\end{array}\right)
$$

which reads

$$
\chi^{\prime}=\mathrm{e}^{-\frac{\theta}{2} \mathbf{i}} \chi \mathrm{e}^{\frac{3 \theta}{2} \gamma_{21}}
$$

and we finally get

$$
\mathbf{D}^{\prime} \Psi^{\prime} \gamma_{012}+m \rho^{\prime} \chi^{\prime}=\mathrm{e}^{-\frac{\theta}{2} \mathbf{i}}\left(\mathbf{D} \Psi \gamma_{012}+m \rho \chi\right) \mathrm{e}^{\frac{3 \theta}{2} \gamma_{21}}=0
$$

We may then say that the wave equation is gauge invariant under the gauge transformation generated by $P_{0}$.

\subsection{Gauge Invariance-Group Generated by $\boldsymbol{P}_{3}$}

This generator acts only upon left waves: we get 


$$
\xi_{1}^{\prime}=\xi_{1} ; \quad \xi_{2}^{\prime}=\xi_{2}
$$

And with left waves we get

$$
\begin{aligned}
& \Psi_{L}^{\prime}=\Psi_{L} \mathrm{e}^{-\theta \mathrm{i}} \\
& \left(\begin{array}{cc}
\phi_{L}^{\prime} & \phi_{n}^{\prime} \\
\hat{\phi}_{n}^{\prime} & \hat{\phi}_{L}^{\prime}
\end{array}\right)=\left(\begin{array}{cc}
\phi_{L} & \phi_{n} \\
\hat{\phi}_{n} & \hat{\phi}_{L}
\end{array}\right)\left(\begin{array}{cc}
\mathrm{e}^{-i \theta} & 0 \\
0 & \mathrm{e}^{i \theta}
\end{array}\right) \\
& \phi_{L}^{\prime}=\mathrm{e}^{-i \theta} \phi_{L} ; \phi_{n}^{\prime}=\mathrm{e}^{i \theta} \phi_{n}
\end{aligned}
$$

That reads

$$
\eta_{1}^{\prime}=\mathrm{e}^{\mathrm{i} \theta} \eta_{1} ; \eta_{2}^{\prime}=\mathrm{e}^{\mathrm{i} \theta} \eta_{2} ; \zeta_{1}^{\prime}=\mathrm{e}^{-i \theta} \zeta_{1} ; \zeta_{2}^{\prime}=\mathrm{e}^{-i \theta} \zeta_{2}
$$

We then get for $a, b, c$ :

$$
\begin{aligned}
& a^{\prime}=\mathrm{e}^{-i \theta} a ; \quad a^{\prime} a^{\prime *}=a a^{*} \\
& b^{\prime}=b ; \quad b^{\prime} b^{\prime *}=b b^{*} \\
& c^{\prime}=\mathrm{e}^{\mathrm{i} \theta} c ; \quad c^{\prime{ }^{\prime *}}{c^{\prime *}}^{*} c c^{*} ; \rho^{\prime}=\rho
\end{aligned}
$$

The covariant derivative is here reduced to

$$
\mathbf{D}=\partial+\frac{g_{2}}{2} \mathbf{W}^{3} P_{3}
$$

We let

$$
\left[\partial \Psi+\frac{g_{2}}{2} \mathbf{W}^{3} P_{3}(\Psi)\right] \gamma_{012}+m \rho \chi=\left(\begin{array}{cc}
A & B \\
\hat{B} & \hat{A}
\end{array}\right) .
$$

We get

$$
\begin{aligned}
& A=\left(\nabla \hat{\phi}_{e}+i \frac{g_{2}}{2} W^{3} \hat{\phi}_{L}\right) \sigma_{21}+\frac{m}{\rho}\left(a^{*} \phi_{e}-b \phi_{n} \sigma_{1}+c^{*} \phi_{n}\right) \\
& B=\left(\nabla \hat{\phi}_{n}-i \frac{g_{2}}{2} W^{3} \hat{\phi}_{n}\right) \sigma_{21}+\frac{m}{\rho}\left(b \phi_{L} \sigma_{1}+c^{*} \phi_{R}\right)
\end{aligned}
$$

Only the left column of $B$ is not null, and the result for $B$ is simple:

$$
B^{\prime}=\mathrm{e}^{-i \theta} B
$$

For $A$, which has a left and a right column, we note:

$$
\begin{aligned}
& A=A_{L}+A_{R} \\
& A_{L}=\left(\nabla \hat{\phi}_{L}+i \frac{g_{2}}{2} W^{3} \hat{\phi}_{L}\right) \sigma_{21}+\frac{m}{\rho}\left(a^{*} \phi_{R}-b \phi_{n} \sigma_{1}\right) \\
& A_{R}=\nabla \hat{\phi}_{R} \sigma_{21}+\frac{m}{\rho}\left(a^{*} \phi_{L}+c^{*} \phi_{n}\right)
\end{aligned}
$$

We then get

$$
A_{L}^{\prime}=\mathrm{e}^{\mathrm{i} \theta} A_{L} ; \quad A_{R}^{\prime}=A_{R} ; \quad A^{\prime}=A\left(\begin{array}{cc}
\mathrm{e}^{\mathrm{i} \theta} & 0 \\
0 & 1
\end{array}\right)
$$

Since the same matrix multiplies the differential part and the mass part of the wave equation, we may say that this equation is invariant under the gauge generated by $P_{3}$. We must remark that, even if the wave has value in the Clifford algebra of space-time, it is much easier to use its components in the Clifford algebra of space to get the gauge invariance. The Clifford algebra of space-time is too much symmetric to be the true frame for a gauge invariance which separates completely left and right waves. 


\subsection{Gauge Invariance-Group Generated by $\boldsymbol{P}_{1}$}

This generator also acts only upon left waves: we get $\xi_{1}^{\prime}=\xi_{1} ; \xi_{2}^{\prime}=\xi_{2}$. And with left waves we get

$$
\begin{aligned}
& \Psi_{L}^{\prime}=\Psi_{L} \mathrm{e}^{\theta \gamma_{3} \mathrm{i}} \\
& \left(\begin{array}{cc}
\phi_{L}^{\prime} & \phi_{n}^{\prime} \\
\hat{\phi}_{n}^{\prime} & \hat{\phi}_{L}^{\prime}
\end{array}\right)=\left(\begin{array}{cc}
\phi_{L} & \phi_{n} \\
\hat{\phi}_{n} & \hat{\phi}_{L}
\end{array}\right)\left(\begin{array}{cc}
\cos (\theta) & -i \sin (\theta) \sigma_{3} \\
-i \sin (\theta) \sigma_{3} & \cos (\theta)
\end{array}\right) \\
& \phi_{L}^{\prime}=\cos (\theta) \phi_{L}-i \sin (\theta) \phi_{n} \sigma_{3} ; \phi_{n}^{\prime}=\cos (\theta) \phi_{n}-i \sin (\theta) \phi_{L} \sigma_{3}
\end{aligned}
$$

which reads with $C=\cos (\theta)$ and $S=\sin (\theta)$

$$
\begin{aligned}
\phi_{L}^{\prime} & =C \phi_{L}+i S \phi_{n} ; \hat{\phi}_{L}^{\prime}=C \hat{\phi}_{L}-i S \hat{\phi}_{n} \\
\phi_{n}^{\prime} & =C \phi_{n}+i S \phi_{L} ; \quad \hat{\phi}_{n}^{\prime}=C \hat{\phi}_{n}-i S \hat{\phi}_{L} \\
\eta_{1}^{\prime} & =C \eta_{1}-i S \zeta_{1} ; \quad \eta_{2}^{\prime}=C \eta_{1}-i S \zeta_{2} \\
\zeta_{1}^{\prime} & =C \zeta_{1}-i S \eta_{1} ; \quad \zeta_{2}^{\prime}=C \zeta_{2}-i S \eta_{2} .
\end{aligned}
$$

We get for $a, b, c$

$$
\begin{aligned}
& a^{\prime}=C a+i S c ; b^{\prime}=b ; \\
& c^{\prime}=C c+i S a \\
& \rho^{\prime 2}=a^{\prime} a^{\prime^{*}}+b^{\prime} b^{\prime^{*}}+c^{\prime} c^{\prime^{*}} \\
& \quad=a a^{*}+b b^{*}+c c^{*}=\rho^{2} .
\end{aligned}
$$

The covariant derivative is now reduced to

$$
\mathbf{D}=\partial+\frac{g_{2}}{2} \mathbf{W}^{1} P_{1}
$$

We let

$$
\left[\partial \Psi+\frac{g_{2}}{2} \mathbf{W}^{1} P_{1}(\Psi)\right] \gamma_{012}+m \rho \chi=\left(\begin{array}{cc}
A & B \\
\hat{B} & \hat{A}
\end{array}\right) .
$$

We get

$$
\begin{aligned}
& A=\nabla \hat{\phi}_{e} \sigma_{21}-\frac{g_{2}}{2} W^{1} \hat{\phi}_{n}+\frac{m}{\rho}\left(a^{*} \phi_{e}-b \phi_{n} \sigma_{1}+c^{*} \phi_{n}\right) \\
& B=\nabla \hat{\phi}_{n} \sigma_{21}-\frac{g_{2}}{2} W^{1} \hat{\phi}_{L}+\frac{m}{\rho}\left(b \phi_{L} \sigma_{1}+c^{*} \phi_{R}\right)
\end{aligned}
$$

As previously, for $A$, which has a left and a right column, we note:

$$
\begin{aligned}
& A=A_{L}+A_{R} \\
& A_{L}=\left(\nabla \hat{\phi}_{L}+i \frac{g_{2}}{2} W^{1} \hat{\phi}_{L}\right) \sigma_{21}+\frac{m}{\rho}\left(a^{*} \phi_{R}-b \phi_{n} \sigma_{1}\right) \\
& A_{R}=\nabla \hat{\phi}_{R} \sigma_{21}+\frac{m}{\rho}\left(a^{*} \phi_{L}+c^{*} \phi_{n}\right)
\end{aligned}
$$

We then get

$$
\begin{aligned}
& A_{L}^{\prime}=C A_{L}-i S B \\
& A_{R}^{\prime}=A_{R} \\
& B^{\prime}=C B-i S A_{L}
\end{aligned}
$$

Since the mass term is changed exactly in the same way that the differential term we can say that this wave equation is gauge invariant under the gauge generated by $P_{1}$. Now it is not necessary to study $P_{2}$, since 
$P_{2}=P_{3} P_{1}$. We have then proved both the form invariance and the gauge invariance of the wave equation (2.43) under the $U(1) \times S U(2)$ Lie group generated by $P_{0}, P_{1}, P_{2}, P_{3}$.

\section{Conservative Current}

We start from (2.36) and its conjugated equation:

$$
0=\hat{\nabla} \phi_{e} \sigma_{21}+\frac{g_{1}}{2} \hat{B}\left(\phi_{e}+\phi_{R}\right)+\frac{g_{2}}{2}\left[\left(-\hat{W}^{1}+i \hat{W}^{2}\right) \phi_{n}+\hat{W}^{3} \phi_{L}\right]+\frac{m}{\rho}\left(a \hat{\phi}_{e}+b^{*} \hat{\phi}_{n} \sigma_{1}+c \hat{\phi}_{n}\right)
$$

and from (2.40) and its conjugated equation

$$
0=\hat{\nabla} \phi_{n} \sigma_{21}+\frac{g_{1}}{2} \hat{B} \phi_{n}+\frac{g_{2}}{2}\left[\left(-\hat{W}^{1}-i \hat{W}^{2}\right) \phi_{L}-\hat{W}^{3} \phi_{n}\right]+\frac{m}{\rho}\left(c \hat{\phi}_{R}-b^{*} \hat{\phi}_{L} \sigma_{1}\right) .
$$

The differential term of (2.44) is

$$
\left(\begin{array}{cc}
\bar{\phi}_{e} & \phi_{n}^{\dagger} \\
\bar{\phi}_{n} & \phi_{e}^{\dagger}
\end{array}\right)\left(\begin{array}{cc}
0 & \nabla \\
\hat{\nabla} & 0
\end{array}\right)\left(\begin{array}{cc}
\phi_{e} & \phi_{n} \\
\hat{\phi}_{n} & \hat{\phi}_{e}
\end{array}\right)\left(\begin{array}{cc}
0 & \sigma_{21} \\
\sigma_{21} & 0
\end{array}\right)=\left(\begin{array}{ll}
\bar{\phi}_{e} \nabla \hat{\phi}_{e} \sigma_{21}+\phi_{n}^{\dagger} \hat{\nabla} \phi_{n} \sigma_{21} & \bar{\phi}_{e} \nabla \hat{\phi}_{n} \sigma_{21}+\phi_{n}^{\dagger} \hat{\nabla} \phi_{e} \sigma_{21} \\
\bar{\phi}_{n} \nabla \hat{\phi}_{e} \sigma_{21}+\phi_{e}^{\dagger} \hat{\nabla} \phi_{n} \sigma_{21} & \bar{\phi}_{n} \nabla \hat{\phi}_{n} \sigma_{21}+\phi_{e}^{\dagger} \hat{\nabla} \phi_{e} \sigma_{21}
\end{array}\right)
$$

Then (2.43) reads $\left(\begin{array}{ll}A & B \\ \hat{B} & \hat{A}\end{array}\right)=0$, which is equivalent to $A=0 ; B=0$. To get the first equation we multiply (2.36) on the left side by $\bar{\phi}_{e}$ and (4.2) by $\phi_{n}^{\dagger}$, this gives

We shall use

$$
\begin{aligned}
0= & \left(\bar{\phi}_{e} \nabla \hat{\phi}_{e}+\phi_{n}^{\dagger} \hat{\nabla} \phi_{n}\right) \sigma_{21}+\frac{g_{1}}{2}\left[\bar{\phi}_{e} B\left(\hat{\phi}_{e}+\hat{\phi}_{R}\right)+\phi_{n}^{\dagger} \hat{B} \phi_{n}\right] \\
& +\frac{g_{2}}{2}\left[-\bar{\phi}_{e}\left(W^{1}+i W^{2}\right) \hat{\phi}_{n}+\bar{\phi}_{e} W^{3} \hat{\phi}_{L}+\phi_{n}^{\dagger}\left(-\hat{W}^{1}-i \hat{W}^{2}\right) \phi_{L}-\phi_{n}^{\dagger} \hat{W}^{3} \phi_{n}\right] \\
& +\frac{m}{\rho}\left[\bar{\phi}_{e}\left(a^{*} \phi_{e}-b \phi_{n} \sigma_{1}+c^{*} \phi_{n}\right)+\phi_{n}^{\dagger}\left(c \hat{\phi}_{R}-b^{*} \hat{\phi}_{L} \sigma_{1}\right)\right]
\end{aligned}
$$

$$
\bar{\phi}_{e} \nabla \hat{\phi}_{e}=\frac{1}{2}\left[\bar{\phi}_{e} \sigma^{\mu}\left(\partial_{\mu} \hat{\phi}_{e}\right)+\left(\partial_{\mu} \bar{\phi}_{e}\right) \sigma^{\mu} \hat{\phi}_{e}\right]+\frac{1}{2}\left[\bar{\phi}_{e} \sigma^{\mu}\left(\partial_{\mu} \hat{\phi}_{e}\right)-\left(\partial_{\mu} \bar{\phi}_{e}\right) \sigma^{\mu} \hat{\phi}_{e}\right]
$$

We got in [1] (A.18), for any space-time vector $A$, the following equality:

$$
\bar{\phi}_{e} A \hat{\phi}_{e}=A_{\nu} D_{\mu}^{v} \sigma^{\mu} ; D_{\mu}=\phi_{e} \sigma_{\mu} \phi_{e}^{\dagger}=D_{\mu}^{v} \sigma_{v} .
$$

Then the first part of (4.5) reads

$$
\frac{1}{2}\left[\bar{\phi}_{e} \sigma^{\mu}\left(\partial_{\mu} \hat{\phi}_{e}\right)+\left(\partial_{\mu} \bar{\phi}_{e}\right) \sigma^{\mu} \hat{\phi}_{e}\right]=\frac{1}{2} \partial_{\mu}\left(\bar{\phi}_{e} \sigma^{\mu} \hat{\phi}_{e}\right)=\frac{1}{2} \partial_{\mu} D_{v}^{\mu} \sigma^{v}=\frac{1}{2}\left(\nabla \cdot D_{v}\right) \sigma^{v}
$$

Since the second part of (4.5) satisfies

$$
\frac{1}{2}\left[\bar{\phi}_{e} \sigma^{\mu}\left(\partial_{\mu} \hat{\phi}_{e}\right)-\left(\partial_{\mu} \bar{\phi}_{e}\right) \sigma^{\mu} \hat{\phi}_{e}\right]^{\dagger}=-\frac{1}{2}\left[\bar{\phi}_{e} \sigma^{\mu}\left(\partial_{\mu} \hat{\phi}_{e}\right)-\left(\partial_{\mu} \bar{\phi}_{e}\right) \sigma^{\mu} \hat{\phi}_{e}\right]
$$

It is a pseudo-vector in space-time and we let

$$
\bar{\phi}_{e} \sigma^{\mu}\left(\partial_{\mu} \hat{\phi}_{e}\right)-\left(\partial_{\mu} \bar{\phi}_{e}\right) \sigma^{\mu} \hat{\phi}_{e}=\left(\nabla \wedge D_{\mu}\right) i \sigma^{\mu} \text {. }
$$

This gives

$$
\bar{\phi}_{e} \nabla \hat{\phi}_{e}=\frac{1}{2}\left(\nabla \cdot D_{\mu}\right) \sigma^{\mu}+\frac{1}{2}\left(\nabla \wedge D_{\mu}\right) i \sigma^{\mu}
$$

Similarly we let

$$
\phi_{n}^{\dagger} \hat{\nabla} \phi_{n}=\frac{1}{2}\left[\left(\nabla \cdot D_{n}\right)+i\left(\nabla \wedge D_{n}\right)\left(1-\sigma_{3}\right)\right]
$$


which gives

$$
\begin{aligned}
2\left(\bar{\phi}_{e} \nabla \hat{\phi}_{e}+\phi_{n}^{\dagger} \hat{\nabla} \phi_{n}\right) \sigma_{21}= & -\nabla \wedge\left(D_{3}+D_{n}\right)-\left(\nabla \cdot D_{2}\right) \sigma_{1}+\left(\nabla \cdot D_{1}\right) \sigma_{2}+\nabla \wedge\left(D_{0}+D_{n}\right) \sigma_{3} \\
& -\left(\nabla \wedge D_{2}\right) i \sigma_{1}+\left(\nabla \wedge D_{1}\right) i \sigma_{2}-\nabla \cdot\left(D_{0}+D_{n}\right) i \sigma_{3}+\nabla \cdot\left(D_{3}+D_{n}\right) i .
\end{aligned}
$$

Next we get, with Appendix A:

$$
\begin{aligned}
& \bar{\phi}_{e} B \hat{\phi}_{e}=\left(B \cdot D_{\mu}\right) \sigma^{\mu}=B \cdot D_{0}-\left(B \cdot D_{1}\right) \sigma_{1}-\left(B \cdot D_{2}\right) \sigma_{2}-\left(B \cdot D_{3}\right) \sigma_{3} \\
& \bar{\phi}_{e} B \hat{\phi}_{R}=\frac{1}{2}\left(-B \cdot D_{1}+i B \cdot D_{2}\right)\left(\sigma_{1}+i \sigma_{2}\right)+\left(B \cdot D_{R}\right)\left(1-\sigma_{3}\right) \\
& \phi_{n}^{\dagger} \hat{B} \phi_{n}=\left(B \cdot D_{n}\right)\left(1-\sigma_{3}\right) .
\end{aligned}
$$

This gives

$$
\begin{aligned}
& \bar{\phi}_{e} B\left(\hat{\phi}_{e}+\hat{\phi}_{R}\right)+\phi_{n}^{\dagger} B \phi_{n}=B \cdot\left(D_{0}+D_{R}+D_{n}\right)-\frac{3}{2}\left(B \cdot D_{1}\right) \sigma_{1} \\
& -\frac{3}{2}\left(B \cdot D_{2}\right) \sigma_{2}-B \cdot\left(D_{3}+D_{R}+D_{n}\right) \sigma_{3}+\frac{1}{2}\left(B \cdot D_{2}\right) i \sigma_{1}-\frac{1}{2}\left(B \cdot D_{1}\right) i \sigma_{2} .
\end{aligned}
$$

We also need

$$
\begin{gathered}
-\bar{\phi}_{e}\left(W^{1}+i W^{2}\right) \hat{\phi}_{n}+\bar{\phi}_{e} W^{3} \hat{\phi}_{L}+\phi_{n}^{\dagger}\left(-\hat{W}^{1}-i \hat{W}^{2}\right) \phi_{L}-\phi_{n}^{\dagger} \hat{W}^{3} \phi_{n} \\
=-\left(\bar{\phi}_{e} W^{1} \hat{\phi}_{n}+\phi_{n}^{\dagger} \hat{W}^{1} \phi_{L}\right)-i\left(\bar{\phi}_{e} W^{2} \hat{\phi}_{n}+\phi_{n}^{\dagger} \hat{W}^{2} \phi_{L}\right)+\left(\bar{\phi}_{e} W^{3} \hat{\phi}_{L}+\phi_{n}^{\dagger} \hat{W}^{3} \phi_{n}\right) .
\end{gathered}
$$

We get with Appendix A:

$$
\begin{gathered}
\bar{\phi}_{e} W^{1} \hat{\phi}_{n}+\phi_{n}^{\dagger} \hat{W}^{1} \phi_{L}=W_{\mu}^{1} C_{6}^{\mu}-\frac{1}{2} W_{\mu}^{1} C_{3}^{\mu}\left(\sigma_{1}-i \sigma_{2}\right) \\
=W_{\mu}^{1}\left(D_{L n}^{\mu}-i d_{L n}^{\mu}\right)-\frac{1}{2} W_{\mu}^{1}\left(D_{R n}^{\mu}+i d_{R n}^{\mu}\right)\left(\sigma_{1}-i \sigma_{2}\right) \\
=W \cdot D_{L n}-\frac{1}{2}\left(W^{1} \cdot D_{R n}\right) \sigma_{1}-\frac{1}{2}\left(W^{1} \cdot d_{R n}\right) \sigma_{2}-\frac{1}{2}\left(W^{1} \cdot d_{R n}\right) i \sigma_{1} \\
+\frac{1}{2}\left(W^{1} \cdot D_{R n}\right) i \sigma_{2}-\left(W^{1} \cdot d_{L n}\right) i .
\end{gathered}
$$

which gives

$$
\begin{gathered}
i\left(\bar{\phi}_{e} W^{2} \hat{\phi}_{n}+\phi_{n}^{\dagger} \hat{W}^{2} \phi_{L}\right)=W^{2} \cdot d_{L n}+\frac{1}{2}\left(W^{2} \cdot d_{R n}\right) \sigma_{1}-\frac{1}{2}\left(W^{2} \cdot D_{R n}\right) \sigma_{2} \\
-\frac{1}{2}\left(W^{2} \cdot D_{R n}\right) i \sigma_{1}-\frac{1}{2}\left(W^{2} \cdot d_{R n}\right) i \sigma_{2}+\left(W^{2} \cdot D_{L n}\right) i
\end{gathered}
$$

We get also with Appendix A:

$$
\begin{aligned}
& \bar{\phi}_{e} W^{3} \hat{\phi}_{L}-\phi_{n}^{\dagger} \hat{W}^{3} \phi_{n}=W_{\mu}^{3} D_{L}^{\mu}\left(1+\sigma_{3}\right)+W_{\mu}^{3} D_{n}^{\mu}\left(-1+\sigma_{3}\right)-\frac{1}{2} W_{\mu}^{1} C_{1}^{\mu}\left(\sigma_{1}-i \sigma_{2}\right) \\
&=W_{\mu}^{3} D_{L}^{\mu}\left(1+\sigma_{3}\right)+W_{\mu}^{3} D_{n}^{\mu}\left(-1+\sigma_{3}\right)-\frac{1}{2} W_{\mu}^{1}\left(D_{1}^{\mu}+i D_{2}^{\mu}\right)\left(\sigma_{1}-i \sigma_{2}\right) \\
&=W^{3} \cdot\left(D_{L}-D_{n}\right)-\frac{1}{2}\left(W^{3} \cdot D_{1}\right) \sigma_{1}-\frac{1}{2}\left(W^{3} \cdot D_{2}\right) \sigma_{2}+\left(W^{3} \cdot\left(D_{L}+D_{n}\right)\right) \sigma_{3} \\
&-\frac{1}{2}\left(W^{3} \cdot D_{2}\right) i \sigma_{1}+\frac{1}{2}\left(W^{3} \cdot D_{1}\right) i \sigma_{2} .
\end{aligned}
$$

Since the mass term satisfies (3.2) the wave equation (4.4) is equivalent to the system: 


$$
\begin{aligned}
& 0=-\nabla \wedge\left(D_{3}+D_{n}\right)+g_{1} B \cdot\left(D_{0}+D_{R}+D_{n}\right)+g_{2}\left[-W^{1} \cdot D_{L n}-W^{2} \cdot d_{L n}+W^{3} \cdot\left(D_{L}-D_{n}\right)\right]+2 m \rho \\
& 0=-\nabla \cdot D_{2}-\frac{3 g_{1}}{2} B \cdot D_{1}+\frac{g_{2}}{2}\left[W^{1} \cdot D_{R n}-W^{2} \cdot d_{R n}-W^{3} \cdot D_{1}\right]-\frac{2 m}{\rho} \mathfrak{R}(b c) \\
& 0=\nabla \cdot D_{1}-\frac{3 g_{1}}{2} B \cdot D_{2}+\frac{g_{2}}{2}\left[W^{1} \cdot d_{R n}+W^{2} \cdot D_{R n}-W^{3} \cdot D_{2}\right]-\frac{2 m}{\rho} \Im(b c) \\
& 0=\nabla \wedge\left(D_{0}+D_{n}\right)-g_{1} B \cdot\left(D_{3}+D_{R}+D_{n}\right)+g_{2} W^{3} \cdot\left(D_{L}+D_{n}\right)-\frac{2 m}{\rho} c c^{*} \\
& 0=-\nabla \wedge D_{2}+\frac{g_{1}}{2} B \cdot D_{2}+\frac{g_{2}}{2}\left(W^{1} \cdot d_{R n}+W^{2} \cdot D_{R n}-W^{3} \cdot D_{2}\right) \\
& 0=\nabla \wedge D_{1}-\frac{g_{1}}{2} B \cdot D_{1}+\frac{g_{2}}{2}\left(-W^{1} \cdot D_{R n}+W^{2} \cdot d_{R n}+W^{3} \cdot D_{1}\right) \\
& 0=-\nabla \cdot\left(D_{0}+D_{n}\right) \\
& 0=\nabla \cdot\left(D_{3}+D_{n}\right)+g_{2}\left(W^{1} \cdot d_{L n}-W^{2} \cdot D_{L n}\right) .
\end{aligned}
$$

A conservative current exists: it is the total $D_{0}+D_{n}$ current where $D_{0}$ is the current of probability in the case of the alone electron, and $D_{n}$ is the isotropic current of the neutrino. Now these currents are not separately conservative, only the total $D_{0}+D_{n}$ current is conservative. The calculation is similar for $B=0$. We get

$$
\begin{aligned}
& 0=-\nabla \wedge d_{L n}+g_{1} B \cdot D_{L n}-g_{2} W^{1} \cdot\left(D_{L}+D_{n}\right)+2 m \frac{a c^{*}}{\rho} \\
& 0=-\nabla \wedge D_{L n}-g_{1} B \cdot d_{L n}+g_{2} W^{2} \cdot\left(D_{L}+D_{n}\right) \\
& 0=-\nabla \cdot d_{L n}-g_{2}\left[W^{1} \cdot\left(D_{L}-D_{n}\right)+W^{3} \cdot D_{L n}\right] \\
& 0=-\nabla \cdot D_{L n}+g_{2}\left[W^{2} \cdot\left(D_{L}-D_{n}\right)+W^{3} \cdot d_{L n}\right] \\
& 0=-\nabla \wedge d_{R n}+\frac{g_{1}}{2} B \cdot D_{R n}+\frac{g_{2}}{2}\left(W \cdot D_{1}+W^{2} \cdot D_{2}+W^{3} \cdot D_{R n}\right) \\
& 0=\nabla \wedge D_{R n}+\frac{g_{1}}{2} B \cdot d_{R n}+\frac{g_{2}}{2}\left(W^{1} \cdot D_{2}-W^{2} \cdot D_{1}+W \cdot d_{R n}\right)
\end{aligned}
$$

\section{Concluding Remarks}

The conservative law $\nabla \cdot\left(D_{0}+D_{n}\right)=0$ is obviously the simplest equation in (4.19). Contrarily to the case of the electron alone, where $D_{3}$ is also a conservative current [4], we get here only one conservative current. When the electron is alone, the conservative $D_{0}$ current is interpreted as the probability density of presence of the electron. It is really the relativistic generalization of the probability density of the Schrödinger wave. But it is nonsense to think the same for the $D_{0}+D_{n}$ current, since we have here both an electron and its neutrino.

The mass term of (2.43) replaces $\rho_{e}$ by $\rho$. This is enough to get a wave equation both form invariant and gauge invariant. To get this important novelty in the electro-weak gauge theory, the use of the Clifford algebra of the physical space is essential. It should be impossible to get this using only the algebra of Dirac matrices or the Clifford algebra of space-time. The physical reason is the difference between left and right waves.

We previously studied [9] a wave equation for the electron, with a mass term similar to the mass term obtained here to account for the electron and its neutrino. Another difference between this study and the present one is the role of the Lagrangian density. In the case of the electron alone, or here, the link between the Lagrangian density and the wave equation is double: the Lagrangian density is obtained from the real part of the invariant wave equation, and the wave equation is obtained from the Lagrange equations. Developing the first equation (4.19) and using (2.25) we can see that the first equation (4.19) is $0=2 \mathcal{L}$. In [9], we studied a case, which could be extended to the present study, where this double link is cut. The Lagrangian density is again obtained from the real part of the invariant wave equation, but the wave equation cannot be obtained from the Lagrange equations. 
The formalism with Dirac matrices ([1] (6.74)) uses $W^{+}=W^{1}+i W^{2}$ and $W^{-}=-W^{1}+i W^{2}$, but we prefer $W^{1}$ and $W^{2}$ : because they are true space-time vectors, because the $i$ term is here not the usual $i$ of quantum fields, generator of the electric gauge, but the $i$ of the chiral gauge. Moreover calculations are more complicated with $W^{ \pm}$.

When we cancel $\phi_{n}$, we get $b=c=0, \rho=\rho_{e}$; the wave equation is reduced to the homogeneous nonlinear equation previously studied [1] [3]. This equation has the Dirac equation as linear approximation and in the case of the $\mathrm{H}$ atom, a set of orthonormal solutions exists with a $\beta$ angle everywhere defined and small. It is exactly the sufficient condition allowing the Dirac equation to approximate our nonlinear homogeneous wave equation. The mass term that we obtained here is also available for the magnetic monopole studied in [1] and [6] [7] [10].

\section{References}

[1] Daviau, C. and Bertrand, J. (2014) New Insights in the Standard Model of Quantum Physics in Clifford Algebra. http://hal.archives-ouvertes.fr/hal-00907848

[2] Daviau, C. and Bertrand, J. (2014) Nouvelle approche du modèle standard de la physique quantique en algèbre de Clifford, JePublie.

[3] Daviau, C. (2013) Invariant Quantum Wave Equations and Double Space-Time. Advances in Imaging and Electron Physics, 179, 1-136. http://dx.doi.org/10.1016/B978-0-12-407700-3.00001-6

[4] Daviau, C. (2012) Nonlinear Dirac Equation, Magnetic Monopoles and Double Space-Time, CISP, Cambridge (UK).

[5] Daviau, C. (2012) Double Space-Time and more, JePublie.

[6] Lochak, G. (1983) Sur un monopôle de masse nulle décrit par l'équation de Dirac et sur une équation générale non linéaire qui contient des monopôles de spin 1/2. Annales de la Fondation Louis de Broglie, 8, 345-370.

[7] Lochak, G. (1985) Wave Equation of a Magnetic Monopole. International Journal of Theoretical Physics, 24, 1019-1050. http://dx.doi.org/10.1007/BF00670815

[8] Daviau, C. (2005) Interprétation cinématique de l’onde de l'électron. Annales de la Fondation Louis de Broglie, 30, 409-428.

[9] Daviau, C. and Bertrand, J. (2013) Annales de la Fondation Louis de Broglie, 38, 57-81.

[10] Lochak, G. (1985) The Symmetry between Electricity and Magnetism and the Wave Equation of a Spin $1 / 2$ Magnetic Monopole. Proceedings of the 4th International Seminar on the Mathematical Theory of Dynamical Systems and Microphysics, Udine, 4-13 September 1985, 107-131. 


\section{Appendix A}

The space-time vector $D_{R}$ satisfies

$$
\begin{aligned}
& D_{R}=\phi_{R} \phi_{R}^{\dagger}=D_{R}^{\mu} \sigma_{\mu}=\left(\begin{array}{cc}
D_{R}^{0}+D_{R}^{3} & D_{R}^{1}-i D_{R}^{2} \\
D_{R}^{1}+i D_{R}^{2} & D_{R}^{0}-D_{R}^{3}
\end{array}\right) \\
& D_{R}^{0}=\xi_{1} \xi_{1}^{*}+\xi_{2} \xi_{2}^{*} ; D_{R}^{1}=\xi_{1} \xi_{2}^{*}+\xi_{2} \xi_{1}^{*} ; D_{R}^{2}=i\left(\xi_{1} \xi_{2}^{*}-\xi_{2} \xi_{1}^{*}\right) ; D_{R}^{3}=\xi_{1} \xi_{1}^{*}-\xi_{2} \xi_{2}^{*}
\end{aligned}
$$

The space-time bivector $S_{R}$ satisfies

$$
\begin{gathered}
S_{R}=\phi_{R} \sigma_{1} \bar{\phi}_{R}=S_{R}^{23} \sigma_{1}+S_{R}^{31} \sigma_{2}+S_{R}^{12} \sigma_{3}+S_{R}^{10} i \sigma_{1}+S_{R}^{20} i \sigma_{2}+S_{R}^{30} i \sigma_{3}=2\left(\begin{array}{cc}
-\xi_{1} \xi_{2} & \xi_{1}^{2} \\
-\xi_{2}^{2} & \xi_{1} \xi_{2}
\end{array}\right) \\
S_{R}^{12}=-\xi_{1} \xi_{2}-\xi_{1}^{*} \xi_{2}^{*} ; S_{R}^{30}=i\left(\xi_{1} \xi_{2}-\xi_{1}^{*} \xi_{2}^{*}\right) ; S_{R}^{23}=\frac{1}{2}\left(\xi_{1}^{2}-\xi_{2}^{2}+\xi_{1}^{* 2}-\xi_{2}^{* 2}\right) \\
S_{R}^{10}=\frac{i}{2}\left(-\xi_{1}^{2}+\xi_{2}^{2}+\xi_{1}^{* 2}-\xi_{2}^{* 2}\right) ; S_{R}^{20}=\frac{1}{2}\left(\xi_{1}^{2}+\xi_{2}^{2}+\xi_{1}^{* 2}+\xi_{2}^{* 2}\right) ; S_{R}^{31}=\frac{i}{2}\left(\xi_{1}^{2}+\xi_{2}^{2}-\xi_{1}^{* 2}-\xi_{2}^{* 2}\right)
\end{gathered}
$$

The space-time vector $D_{n}$ satisfies

$$
\begin{aligned}
& D_{n}=\phi_{n} \phi_{n}^{\dagger}=D_{n}^{\mu} \sigma_{\mu}=\left(\begin{array}{cc}
D_{n}^{0}+D_{n}^{3} & D_{n}^{1}-i D_{n}^{2} \\
D_{n}^{1}+i D_{n}^{2} & D_{n}^{0}-D_{n}^{3}
\end{array}\right) \\
& D_{n}^{0}=\zeta_{1} \zeta_{1}^{*}+\zeta_{2} \zeta_{2}^{*} ; D_{n}^{1}=-\zeta_{1} \zeta_{2}^{*}-\zeta_{2} \zeta_{1}^{*} ; D_{n}^{2}=i\left(-\zeta_{1} \zeta_{2}^{*}+\zeta_{2} \zeta_{1}^{*}\right) ; D_{n}^{3}=-\zeta_{1} \zeta_{1}^{*}+\zeta_{2} \zeta_{2}^{*}
\end{aligned}
$$

The space-time bivector $S_{n}$ satisfies

$$
\begin{gathered}
S_{n}=\phi_{n} \sigma_{1} \bar{\phi}_{n}=S_{n}^{23} \sigma_{1}+S_{n}^{31} \sigma_{2}+S_{n}^{12} \sigma_{3}+S_{n}^{10} i \sigma_{1}+S_{n}^{20} i \sigma_{2}+S_{n}^{30} i \sigma_{3}=2\left(\begin{array}{cc}
-\zeta_{1}^{*} \zeta_{2}^{*} & -\zeta_{2}^{* 2} \\
\zeta_{1}^{* 2} & \zeta_{1}^{*} \zeta_{2}^{*}
\end{array}\right) \\
S_{n}^{12}=-\zeta_{1} \zeta_{2}-\zeta_{1}^{*} \zeta_{2}^{*} ; S_{n}^{30}=-i \zeta_{1} \zeta_{2}+i \zeta_{1}^{*} \zeta_{2}^{*} ; S_{n}^{23}=\frac{1}{2}\left(\zeta_{1}^{2}-\zeta_{2}^{2}+\zeta_{1}^{* 2}-\zeta_{2}^{* 2}\right) \\
S_{n}^{10}=\frac{i}{2}\left(\zeta_{1}^{2}-\zeta_{2}^{2}-\zeta_{1}^{* 2}+\zeta_{2}^{* 2}\right) ; S_{n}^{20}=\frac{1}{2}\left(-\zeta_{1}^{2}-\zeta_{2}^{2}-\zeta_{1}^{* 2}-\zeta_{2}^{* 2}\right) ; S_{n}^{31}=\frac{i}{2}\left(\zeta_{1}^{2}+\zeta_{2}^{2}-\zeta_{1}^{* 2}-\zeta_{2}^{* 2}\right)
\end{gathered}
$$

The space-time vector $D_{L}$ satisfies

$$
\begin{aligned}
& D_{L}=\phi_{L} \phi_{L}^{\dagger}=D_{L}^{\mu} \sigma_{\mu}=\left(\begin{array}{cc}
D_{L}^{0}+D_{L}^{3} & D_{L}^{1}-i D_{L}^{2} \\
D_{L}^{1}+i D_{L}^{2} & D_{L}^{0}-D_{L}^{3}
\end{array}\right) \\
& D_{L}^{0}=\eta_{1} \eta_{1}^{*}+\eta_{2} \eta_{2}^{*} ; \quad D_{L}^{1}=-\eta_{1} \eta_{2}^{*}-\eta_{2} \eta_{1}^{*} ; D_{L}^{2}=i\left(-\eta_{1} \eta_{2}^{*}+\eta_{2} \eta_{1}^{*}\right) ; D_{L}^{3}=-\eta_{1} \eta_{1}^{*}+\eta_{2} \eta_{2}^{*}
\end{aligned}
$$

The space-time bivector $S_{L}$ satisfies

$$
\begin{aligned}
& S_{L}=\phi_{L} \sigma_{1} \bar{\phi}_{L}=S_{L}^{23} \sigma_{1}+S_{L}^{31} \sigma_{2}+S_{L}^{12} \sigma_{3}+S_{L}^{10} i \sigma_{1}+S_{L}^{20} i \sigma_{2}+S_{L}^{30} i \sigma_{3}=2\left(\begin{array}{cc}
-\eta_{1}^{*} \eta_{2}^{*} & -\eta_{2}^{* 2} \\
\eta_{1}^{* 2} & \eta_{1}^{*} \eta_{2}^{*}
\end{array}\right) \\
& S_{L}^{12}=-\eta_{1} \eta_{2}-\eta_{1}^{*} \eta_{2}^{*} ; \quad S_{L}^{30}=-i \eta_{1} \eta_{2}+i \eta_{1}^{*} \eta_{2}^{*} ; S_{L}^{23}=\frac{1}{2}\left(\eta_{1}^{2}-\eta_{2}^{2}+\eta_{1}^{* 2}-\eta_{2}^{* 2}\right) \\
& S_{L}^{10}=\frac{i}{2}\left(\eta_{1}^{2}-\eta_{2}^{2}-\eta_{1}^{* 2}+\eta_{2}^{* 2}\right) ; \quad S_{L}^{20}=\frac{1}{2}\left(-\eta_{1}^{2}-\eta_{2}^{2}-\eta_{1}^{* 2}-\eta_{2}^{* 2}\right) ; \quad S_{L}^{31}=\frac{i}{2}\left(\eta_{1}^{2}+\eta_{2}^{2}-\eta_{1}^{* 2}-\eta_{2}^{* 2}\right)
\end{aligned}
$$

From (1.8) we get (1.9) and

$$
\begin{aligned}
& S_{R L}=\phi_{R} \bar{\phi}_{L}-\phi_{L} \bar{\phi}_{R} \\
& D_{R L}=\phi_{R} \sigma_{1} \phi_{L}^{\dagger}+\phi_{L} \sigma_{1} \phi_{R}^{\dagger} \\
& i d_{R L}=\phi_{R} \sigma_{1} \phi_{L}^{\dagger}-\phi_{L} \sigma_{1} \phi_{R}^{\dagger}
\end{aligned}
$$

\section{Next we use}




$$
\phi_{e} \sigma_{3}=\sqrt{2}\left(\begin{array}{cc}
\xi_{1} & -\eta_{2}^{*} \\
\xi_{2} & \eta_{1}^{*}
\end{array}\right)\left(\begin{array}{cc}
1 & 0 \\
0 & -1
\end{array}\right)=\sqrt{2}\left(\begin{array}{cc}
\xi_{1} & \eta_{2}^{*} \\
\xi_{2} & -\eta_{1}^{*}
\end{array}\right)=\phi_{R}-\phi_{L}
$$

And since $\phi_{R} \bar{\phi}_{R}=0$ and $\phi_{L} \bar{\phi}_{L}=0$ we get from (2.60) in [1]:

$$
S_{3}=\phi_{e} \sigma_{3} \bar{\phi}_{e}=\left(\phi_{R}-\phi_{L}\right)\left(\bar{\phi}_{R}+\bar{\phi}_{L}\right)=\phi_{R} \bar{\phi}_{L}-\phi_{L} \bar{\phi}_{R}=S_{R L} \text {. }
$$

We have

$$
\begin{aligned}
& \phi_{R} \sigma_{1} \phi_{L}^{\dagger}=2\left(\begin{array}{cc}
\xi_{1} & 0 \\
\xi_{2} & 0
\end{array}\right)\left(\begin{array}{ll}
0 & 1 \\
1 & 0
\end{array}\right)\left(\begin{array}{cc}
0 & 0 \\
-\eta_{2} & \eta_{1}
\end{array}\right)=2\left(\begin{array}{cc}
-\xi_{1} \eta_{2} & \xi_{1} \eta_{1} \\
-\xi_{2} \eta_{2} & \xi_{2} \eta_{1}
\end{array}\right) \\
& \phi_{L} \sigma_{1} \phi_{R}^{\dagger}=2\left(\begin{array}{cc}
0 & -\eta_{2}^{*} \\
0 & \eta_{1}^{*}
\end{array}\right)\left(\begin{array}{ll}
0 & 1 \\
1 & 0
\end{array}\right)\left(\begin{array}{cc}
\xi_{1}^{*} & \xi_{2}^{*} \\
0 & 0
\end{array}\right)=2\left(\begin{array}{cc}
-\xi_{1}^{*} \eta_{2}^{*} & -\xi_{2}^{*} \eta_{2}^{*} \\
\xi_{1}^{*} \eta_{1}^{*} & \xi_{2}^{*} \eta_{1}^{*}
\end{array}\right)
\end{aligned}
$$

and with (5.10) we get

$$
\begin{aligned}
D_{R L} & =\left(\begin{array}{cc}
D_{R L}^{0}+D_{R L}^{3} & D_{R L}^{1}-i D_{R L}^{2} \\
D_{R L}^{1}+i D_{R L}^{2} & D_{R L}^{0}-D_{R L}^{3}
\end{array}\right)=2\left(\begin{array}{ll}
-\xi_{1} \eta_{2}-\xi_{1}^{*} \eta_{2}^{*} & \xi_{1} \eta_{1}-\xi_{2}^{*} \eta_{2}^{*} \\
-\xi_{2} \eta_{2}+\xi_{1}^{*} \eta_{1}^{*} & \xi_{2} \eta_{1}+\xi_{2}^{*} \eta_{1}^{*}
\end{array}\right) \\
i d_{R L} & =i\left(\begin{array}{cc}
d_{R L}^{0}+d_{R L}^{3} & d_{R L}^{1}-i d_{R L}^{2} \\
d_{R L}^{1}+i d_{R L}^{2} & d_{R L}^{0}-d_{R L}^{3}
\end{array}\right)=2\left(\begin{array}{ll}
-\xi_{1} \eta_{2}+\xi_{1}^{*} \eta_{2}^{*} & \xi_{1} \eta_{1}+\xi_{2}^{*} \eta_{2}^{*} \\
-\xi_{2} \eta_{2}-\xi_{1}^{*} \eta_{1}^{*} & \xi_{2} \eta_{1}-\xi_{2}^{*} \eta_{1}^{*}
\end{array}\right)
\end{aligned}
$$

which gives

$$
\begin{gathered}
D_{R L}^{0}=-\xi_{1} \eta_{2}+\xi_{2} \eta_{1}-\xi_{1}^{*} \eta_{2}^{*}+\xi_{2}^{*} \eta_{1}^{*} ; D_{R L}^{1}=\xi_{1} \eta_{1}-\xi_{2} \eta_{2}+\xi_{1}^{*} \eta_{1}^{*}-\xi_{2}^{*} \eta_{2}^{*} \\
D_{R L}^{2}=i\left(\xi_{1} \eta_{1}+\xi_{2} \eta_{2}-\xi_{1}^{*} \eta_{1}^{*}-\xi_{2}^{*} \eta_{2}^{*}\right) ; \quad D_{R L}^{3}=-\xi_{1} \eta_{2}-\xi_{2} \eta_{1}-\xi_{1}^{*} \eta_{2}^{*}-\xi_{2}^{*} \eta_{1}^{*} \\
d_{R L}^{0}=i\left(\xi_{1} \eta_{2}-\xi_{2} \eta_{1}-\xi_{1}^{*} \eta_{2}^{*}+\xi_{2}^{*} \eta_{1}^{*}\right) ; d_{R L}^{1}=i\left(-\xi_{1} \eta_{1}+\xi_{2} \eta_{2}+\xi_{1}^{*} \eta_{1}^{*}-\xi_{2}^{*} \eta_{2}^{*}\right) \\
d_{R L}^{2}=\xi_{1} \eta_{1}+\xi_{2} \eta_{2}+\xi_{1}^{*} \eta_{1}^{*}+\xi_{2}^{*} \eta_{2}^{*} ; \quad d_{R L}^{3}=i\left(\xi_{1} \eta_{2}+\xi_{2} \eta_{1}-\xi_{1}^{*} \eta_{2}^{*}-\xi_{2}^{*} \eta_{1}^{*}\right)
\end{gathered}
$$

Comparison with (A.17) to (A.20) and with (A.23) to (A.26) in [5] proves that

$$
D_{R L}=D_{1}=\phi_{e} \sigma_{1} \phi_{e}^{\dagger} ; d_{R L}=D_{2}=\phi_{e} \sigma_{2} \phi_{e}^{\dagger} \text {. }
$$

We let

$$
C_{1}=D_{R L}+i d_{R L} ; C_{2}=D_{R L}-i d_{R L}
$$

which gives

$$
\begin{array}{ll}
C_{1}^{0}=2\left(-\xi_{1} \eta_{2}+\xi_{2} \eta_{1}\right) ; & C_{2}^{0}=2\left(-\xi_{1}^{*} \eta_{2}^{*}+\xi_{2}^{*} \eta_{1}^{*}\right) \\
C_{1}^{1}=2\left(+\xi_{1} \eta_{1}-\xi_{2} \eta_{2}\right) ; \quad C_{2}^{1}=2\left(+\xi_{1}^{*} \eta_{1}^{*}-\xi_{2}^{*} \eta_{2}^{*}\right) \\
C_{1}^{2}=2 i\left(+\xi_{1} \eta_{1}+\xi_{2} \eta_{2}\right) ; \quad C_{2}^{2}=2 i\left(-\xi_{1}^{*} \eta_{1}^{*}-\xi_{2}^{*} \eta_{2}^{*}\right) \\
C_{1}^{3}=2\left(-\xi_{1} \eta_{2}-\xi_{2} \eta_{1}\right) ; \quad C_{2}^{3}=2\left(-\xi_{1}^{*} \eta_{2}^{*}-\xi_{2}^{*} \eta_{1}^{*}\right)
\end{array}
$$

Next from (1.17) we get (1.18) and

$$
S_{R n}=\phi_{R} \bar{\phi}_{n}-\phi_{n} \bar{\phi}_{R}=2\left(\begin{array}{cc}
\xi_{1} \zeta_{1}^{*}-\xi_{2} \zeta_{2}^{*} & 2 \xi_{1} \zeta_{2}^{*} \\
2 \xi_{2} \zeta_{1}^{*} & -\left(\xi_{1} \zeta_{1}^{*}-\xi_{2} \zeta_{2}^{*}\right)
\end{array}\right)
$$

which gives, with notations similar to (5.5)

$$
\begin{aligned}
& S_{R n}^{12}=\xi_{1} \zeta_{1}^{*}-\xi_{2} \zeta_{2}^{*}+\xi_{1}^{*} \zeta_{1}-\xi_{2}^{*} \zeta_{2} ; S_{R n}^{23}=\xi_{1} \zeta_{2}^{*}+\xi_{2} \zeta_{1}^{*}+\xi_{2}^{*} \zeta_{1}+\xi_{1}^{*} \zeta_{2} \\
& S_{R n}^{31}=i\left(\xi_{1} \zeta_{2}^{*}-\xi_{2} \zeta_{1}^{*}+\xi_{2}^{*} \zeta_{1}-\xi_{1}^{*} \zeta_{2}\right) ; S_{R n}^{10}=i\left(-\xi_{1} \zeta_{2}^{*}-\xi_{2} \zeta_{1}^{*}+\xi_{2}^{*} \zeta_{1}+\xi_{1}^{*} \zeta_{2}\right) \\
& S_{R n}^{20}=\xi_{1} \zeta_{2}^{*}-\xi_{2} \zeta_{1}^{*}-\xi_{2}^{*} \zeta_{1}+\xi_{1}^{*} \zeta_{2} ; S_{R n}^{30}=i\left(-\xi_{1} \zeta_{1}^{*}+\xi_{2} \zeta_{2}^{*}+\xi_{1}^{*} \zeta_{1}-\xi_{2}^{*} \zeta_{2}\right)
\end{aligned}
$$


We have

$$
\begin{aligned}
& \phi_{R} \sigma_{1} \phi_{n}^{\dagger}=2\left(\begin{array}{cc}
\xi_{1} & 0 \\
\xi_{2} & 0
\end{array}\right)\left(\begin{array}{ll}
0 & 1 \\
1 & 0
\end{array}\right)\left(\begin{array}{cc}
0 & 0 \\
-\zeta_{2} & \zeta_{1}
\end{array}\right)=2\left(\begin{array}{cc}
-\xi_{1} \zeta_{2} & \xi_{1} \zeta_{1} \\
-\xi_{2} \zeta_{2} & \xi_{2} \zeta_{1}
\end{array}\right) \\
& \phi_{n} \sigma_{1} \phi_{R}^{\dagger}=2\left(\begin{array}{cc}
0 & -\zeta_{2}^{*} \\
0 & \zeta_{1}^{*}
\end{array}\right)\left(\begin{array}{ll}
0 & 1 \\
1 & 0
\end{array}\right)\left(\begin{array}{cc}
\xi_{1}^{*} & \xi_{2}^{*} \\
0 & 0
\end{array}\right)=2\left(\begin{array}{cc}
-\xi_{1}^{*} \zeta_{2}^{*} & -\xi_{2}^{*} \zeta_{2}^{*} \\
\xi_{1}^{*} \zeta_{1}^{*} & \xi_{2}^{*} \zeta_{1}^{*}
\end{array}\right)
\end{aligned}
$$

and with (1.17) we get

$$
\begin{aligned}
D_{R n} & =\left(\begin{array}{ll}
D_{R n}^{0}+D_{R n}^{3} & D_{R n}^{1}-i D_{R n}^{2} \\
D_{R n}^{1}+i D_{R n}^{2} & D_{R n}^{0}-D_{R n}^{3}
\end{array}\right)=2\left(\begin{array}{ll}
-\xi_{1} \zeta_{2}-\xi_{1}^{*} \zeta_{2}^{*} & \xi_{1} \zeta_{1}-\xi_{2}^{*} \zeta_{2}^{*} \\
-\xi_{2} \zeta_{2}+\xi_{1}^{*} \zeta_{1}^{*} & \xi_{2} \zeta_{1}+\xi_{2}^{*} \zeta_{1}^{*}
\end{array}\right) \\
i d_{R n} & =i\left(\begin{array}{ll}
d_{R n}^{0}+d_{R n}^{3} & d_{R n}^{1}-i d_{R n}^{2} \\
d_{R n}^{1}+i d_{R n}^{2} & d_{R n}^{0}-d_{R n}^{3}
\end{array}\right)=2\left(\begin{array}{ll}
-\xi_{1} \zeta_{2}+\xi_{1}^{*} \zeta_{2}^{*} & \xi_{1} \zeta_{1}+\xi_{2}^{*} \zeta_{2}^{*} \\
-\xi_{2} \zeta_{2}-\xi_{1}^{*} \zeta_{1}^{*} & \xi_{2} \zeta_{1}-\xi_{2}^{*} \zeta_{1}^{*}
\end{array}\right)
\end{aligned}
$$

which gives

$$
\begin{gathered}
D_{R n}^{0}=-\xi_{1} \zeta_{2}+\xi_{2} \zeta_{1}-\xi_{1}^{*} \zeta_{2}^{*}+\xi_{2}^{*} \zeta_{1}^{*} ; \quad D_{R n}^{1}=\xi_{1} \zeta_{1}-\xi_{2} \zeta_{2}+\xi_{1}^{*} \zeta_{1}^{*}-\xi_{2}^{*} \zeta_{2}^{*} \\
D_{R n}^{2}=i\left(\xi_{1} \zeta_{1}+\xi_{2} \zeta_{2}-\xi_{1}^{*} \zeta_{1}^{*}-\xi_{2}^{*} \zeta_{2}^{*}\right) ; \quad D_{R n}^{3}=-\xi_{1} \zeta_{2}-\xi_{2} \zeta_{1}-\xi_{1}^{*} \zeta_{2}^{*}-\xi_{2}^{*} \zeta_{1}^{*} \\
d_{R n}^{0}=i\left(\xi_{1} \zeta_{2}-\xi_{2} \zeta_{1}-\xi_{1}^{*} \zeta_{2}^{*}+\xi_{2}^{*} \zeta_{1}^{*}\right) ; \quad d_{R n}^{1}=i\left(-\xi_{1} \zeta_{1}+\xi_{2} \zeta_{2}+\xi_{1}^{*} \zeta_{1}^{*}-\xi_{2}^{*} \zeta_{2}^{*}\right) \\
d_{R n}^{2}=\xi_{1} \zeta_{1}+\xi_{2} \zeta_{2}+\xi_{1}^{*} \zeta_{1}^{*}+\xi_{2}^{*} \zeta_{2}^{*} ; \quad d_{R n}^{3}=i\left(\xi_{1} \zeta_{2}+\xi_{2} \zeta_{1}-\xi_{1}^{*} \zeta_{2}^{*}-\xi_{2}^{*} \zeta_{1}^{*}\right)
\end{gathered}
$$

We let

$$
C_{3}=D_{R n}+i d_{R n} ; \quad C_{4}=D_{R n}-i d_{R n}
$$

which gives

$$
\begin{array}{ll}
C_{3}^{0}=2\left(-\xi_{1} \zeta_{2}+\xi_{2} \zeta_{1}\right) ; & C_{4}^{0}=2\left(-\xi_{1}^{*} \zeta_{2}^{*}+\xi_{2}^{*} \zeta_{1}^{*}\right) \\
C_{3}^{1}=2\left(+\xi_{1} \zeta_{1}-\xi_{2} \zeta_{2}\right) ; & C_{4}^{1}=2\left(+\xi_{1}^{*} \zeta_{1}^{*}-\xi_{2}^{*} \zeta_{2}^{*}\right) \\
C_{3}^{2}=2 i\left(+\xi_{1} \zeta_{1}+\xi_{2} \zeta_{2}\right) ; & C_{4}^{2}=2 i\left(-\xi_{1}^{*} \zeta_{1}^{*}-\xi_{2}^{*} \zeta_{2}^{*}\right) \\
C_{3}^{3}=2\left(-\xi_{1} \zeta_{2}-\xi_{2} \zeta_{1}\right) ; & C_{4}^{3}=2\left(-\xi_{1}^{*} \zeta_{2}^{*}-\xi_{2}^{*} \zeta_{1}^{*}\right)
\end{array}
$$

Finally from (1.15) we get (1.16) and

$$
\begin{aligned}
S_{L n} & =\hat{\phi}_{n} \sigma_{1} \phi_{L}^{\dagger}+\hat{\phi}_{L} \sigma_{1} \phi_{n}^{\dagger} \\
& =2\left(\begin{array}{cc}
-\eta_{1} \zeta_{2}-\eta_{2} \zeta_{1} & 2 \eta_{1} \zeta_{1} \\
-2 \eta_{2} \zeta_{2} & \eta_{1} \zeta_{1}+\eta_{2} \zeta_{2}
\end{array}\right)
\end{aligned}
$$

which gives, with notations similar to (5.5)

$$
\begin{aligned}
& S_{L n}^{12}=-\eta_{1} \zeta_{2}-\eta_{2} \zeta_{1}+\eta_{1}^{*} \zeta_{2}^{*}-\eta_{2}^{*} \zeta_{1}^{*} ; \quad S_{L n}^{23}=\eta_{1} \zeta_{1}-\eta_{2} \zeta_{2}+\eta_{1}^{*} \zeta_{1}^{*}-\eta_{2}^{*} \zeta_{2}^{*} \\
& S_{L n}^{31}=i\left(\eta_{1} \zeta_{1}+\eta_{2} \zeta_{2}-\eta_{1}^{*} \zeta_{1}^{*}-\eta_{2}^{*} \zeta_{2}^{*}\right) ; \quad S_{L n}^{10}=i\left(-\eta_{1} \zeta_{1}+\eta_{2} \zeta_{2}+\eta_{1}^{*} \zeta_{1}^{*}-\eta_{2}^{*} \zeta_{2}^{*}\right) \\
& S_{L n}^{20}=\eta_{1} \zeta_{1}+\eta_{2} \zeta_{2}+\eta_{1}^{*} \zeta_{1}^{*}+\eta_{2}^{*} \zeta_{2}^{*} ; \quad S_{L n}^{30}=i\left(\eta_{1} \zeta_{2}+\eta_{2} \zeta_{1}-\eta_{1}^{*} \zeta_{2}^{*}-\eta_{2}^{*} \zeta_{1}^{*}\right)
\end{aligned}
$$

We have

$$
\begin{aligned}
& \phi_{L} \phi_{n}^{\dagger}=2\left(\begin{array}{cc}
\eta_{2}^{*} \zeta_{2} & -\eta_{2}^{*} \zeta_{1} \\
-\eta_{1}^{*} \zeta_{2} & \eta_{1}^{*} \zeta_{1}
\end{array}\right) ; \\
& \phi_{n} \phi_{L}^{\dagger}=2\left(\begin{array}{cc}
\eta_{2} \zeta_{2}^{*} & -\eta_{1} \zeta_{2}^{*} \\
-\eta_{2} \zeta_{1}^{*} & \eta_{1} \zeta_{1}^{*}
\end{array}\right)
\end{aligned}
$$

and with (1.15) we get 


$$
\begin{aligned}
D_{L n} & =\left(\begin{array}{ll}
D_{L n}^{0}+D_{L n}^{3} & D_{L n}^{1}-i D_{L n}^{2} \\
D_{L n}^{1}+i D_{L n}^{2} & D_{L n}^{0}-D_{L n}^{3}
\end{array}\right)=2\left(\begin{array}{cc}
\eta_{2} \zeta_{2}^{*}+\eta_{2}^{*} \zeta_{2} & -\eta_{1} \zeta_{2}^{*}-\eta_{2}^{*} \zeta_{1} \\
-\eta_{1}^{*} \zeta_{2}-\eta_{2} \zeta_{1}^{*} & \eta_{1} \zeta_{1}^{*}+\eta_{1}^{*} \zeta_{1}
\end{array}\right) \\
i d_{L n} & =i\left(\begin{array}{ll}
d_{L n}^{0}+d_{L n}^{3} & d_{L n}^{1}-i d_{L n}^{2} \\
d_{L n}^{1}+i d_{L n}^{2} & d_{L n}^{0}-d_{L n}^{3}
\end{array}\right)=2\left(\begin{array}{cc}
\eta_{2} \zeta_{2}^{*}-\eta_{2}^{*} \zeta_{2} & -\eta_{1} \zeta_{2}^{*}+\eta_{2}^{*} \zeta_{1} \\
-\eta_{1}^{*} \zeta_{2}+\eta_{2} \zeta_{1}^{*} & \eta_{1} \zeta_{1}^{*}-\eta_{1}^{*} \zeta_{1}
\end{array}\right)
\end{aligned}
$$

which gives

$$
\begin{aligned}
& D_{L n}^{0}=\eta_{1} \zeta_{1}^{*}+\eta_{2} \zeta_{2}^{*}+\eta_{1}^{*} \zeta_{1}+\eta_{2}^{*} \zeta_{2} ; \quad D_{L n}^{1}=-\eta_{2} \zeta_{1}^{*}-\eta_{1} \zeta_{2}^{*}-\eta_{1}^{*} \zeta_{2}-\eta_{2}^{*} \zeta_{1} \\
& D_{L n}^{2}=i\left(\eta_{2} \zeta_{1}^{*}-\eta_{1} \zeta_{2}^{*}+\eta_{1}^{*} \zeta_{2}-\eta_{2}^{*} \zeta_{1}\right) ; \quad D_{L n}^{3}=-\eta_{1} \zeta_{1}^{*}+\eta_{2} \zeta_{2}^{*}-\eta_{1}^{*} \zeta_{1}+\eta_{2}^{*} \zeta_{2} \\
& d_{L n}^{0}=i\left(-\eta_{1} \zeta_{1}^{*}-\eta_{2} \zeta_{2}^{*}+\eta_{1}^{*} \zeta_{1}+\eta_{2}^{*} \zeta_{2}\right) ; \quad d_{L n}^{1}=i\left(\eta_{2} \zeta_{1}^{*}+\eta_{1} \zeta_{2}^{*}-\eta_{1}^{*} \zeta_{2}-\eta_{2}^{*} \zeta_{1}\right) \\
& d_{L n}^{2}=\eta_{2} \zeta_{1}^{*}-\eta_{1} \zeta_{2}^{*}-\eta_{1}^{*} \zeta_{2}+\eta_{2}^{*} \zeta_{1} ; \quad d_{L n}^{3}=i\left(-\eta_{1} \zeta_{1}^{*}+\eta_{2} \zeta_{2}^{*}+\eta_{1}^{*} \zeta_{1}-\eta_{2}^{*} \zeta_{2}\right)
\end{aligned}
$$

We let

$$
C_{5}=D_{L n}+i d_{L n} ; \quad C_{6}=D_{L n}-i d_{L n}
$$

which gives

$$
\begin{aligned}
& C_{5}^{0}=2\left(\eta_{1} \zeta_{1}^{*}+\eta_{1} \zeta_{1}^{*}\right) ; \quad C_{6}^{0}=2\left(\eta_{1}^{*} \zeta_{1}+\eta_{2}^{*} \zeta_{2}\right) \\
& C_{5}^{1}=2\left(-\eta_{2} \zeta_{1}^{*}-\eta_{1} \zeta_{2}^{*}\right) ; \quad C_{6}^{1}=2\left(-\eta_{1}^{*} \zeta_{2}-\eta_{2}^{*} \zeta_{1}\right) \\
& C_{5}^{2}=2 i\left(\eta_{2} \zeta_{1}^{*}-\eta_{1} \zeta_{2}^{*}\right) ; \quad C_{6}^{2}=2 i\left(\eta_{1}^{*} \zeta_{2}-\eta_{2}^{*} \zeta_{1}\right) \\
& C_{5}^{3}=2\left(-\eta_{1} \zeta_{1}^{*}+\eta_{2} \zeta_{2}^{*}\right) ; \quad C_{6}^{3}=2\left(-\eta_{1}^{*} \zeta_{1}+\eta_{2}^{*} \zeta_{2}\right)
\end{aligned}
$$

With $3 \times 2$ scalars, 9 vectors and 6 bivectors, we obtained the $78=3 \times 2+9 \times 4+6 \times 6$ awaited densities. 